\author{
钠离子电池钴酸钠正极材料研究进展 \\ 谢佶晟 ${ }^{a}$ 肖竹梅 ${ }^{a}$ 左文华 $a$ 杨勇*,a,b \\ ( ${ }^{a}$ 厦门大学化学化工学院 固体表面物理化学国家重点实验室＼cjkstart厦门 361005) \\ ( ${ }^{b}$ 厦门大学能源学院 厦门 361102)
}

\begin{abstract}
摘要 钠离子电池凭借分布广泛和低成本的钠资源在大规模电化学能量存储领域受到广泛关注. 层状过渡金属氧化物 作为一种重要的钠离子电池正极材料, 具有比容量高、电化学可逆性相对较好和化学组成丰富且可调的特征, 得到广 泛关注. 其中钴酸钠是一种典型层状过渡金属氧化物, 自 20 世纪 80 年代就得到大量研究. 由于钴酸钠含有丰富的电化 学信息, 基于其充放电过程进行的机理研究对理解钠离子电池层状氧化物体系具有重要意义. 因此在介绍钴酸钠的常 见结构类型与合成相图的基础上, 本文着重综述了不同结构钴酸钠在充放电过程中结构变化和电荷补偿机理的研究进 展, 同时讨论了上述机制对电化学性能的影响. 本综述旨在为深入研究并建立层状过渡金属氧化物正极材料电化学过 程中的构效关系提供支持.
\end{abstract}

关键词＼cjkstart钠离子电池；正极材料；钴酸钠; 结构变化; 电荷补偿机理；构效关系

\title{
Research Progresses of Sodium Cobalt Oxide as Cathode in Sodium Ion Batteries
}

\author{
Jisheng Xie $^{a} \quad$ Zhumei Xiao $^{a} \quad$ Wenhua Zuo $^{a} \quad$ Yong Yang $^{*, a, b}$ \\ ( ${ }^{a}$ State Key Laboratory for Physical Chemistry of Solid Surfaces, College of Chemistry and Chemical Engineering, \\ Xiamen University, Xiamen 361005, China) \\ ( ${ }^{b}$ School of Energy Research, Xiamen University, Xiamen 361102, China)
}

\begin{abstract}
Sodium ion batteries have regained widespread attention in the field of large-scale electrochemical energy storage by virtue of their widely distributed and low-cost sodium resources. Among many of the cathode materials (layered, tunnel-like, polyanionic type and Prussian-blue type, etc.), layered transition metal oxide materials have received extensive research attention due to the features of high specific capacity, relatively good electrochemical reversibility, and rich and adjustable chemical composition. Sodium cobalt oxide is one of the most typical layered transition metal oxides. A lot of research has been done about sodium cobalt oxide since the 1980s. Although compared with other energy storage systems (such as lithium cobalt oxide materials which has the similar composition), sodium cobalt oxide does not take more advantage in electrochemical performance (like rate performance, cycle performance, etc.), but it can be observed from the charge and discharge curve that sodium cobalt oxide has undergone complex electrochemical processes, which means that it has a bunch of information on the degradation mechanisms during the charge and discharge processes. The correlation studies of the failure mechanism during the charging and discharging processes of sodium cobalt oxide (including the structure changes and the charge compensation mechanisms) are of great significance for the deep understanding of the layered oxide systems in sodium ion batteries. Therefore, on the basis of introducing the common crystal structure types and the synthesis phase diagram of sodium cobalt oxides, this article focuses on reviewing the structure changes (including phase transition and $\mathrm{Na}^{+} /$ vacancy ordering) and charge compensation mechanisms of sodium cobalt oxides with different crystal structures during the charging and discharging. At the same time, the correlation between the mechanisms above and electrochemical performance is discussed. This review aims to provide support for the in-depth study and establishment of the structure-activity relationship in the electrochemical processes of layered transition metal oxide cathode materials.
\end{abstract}

Keywords sodium ion battery; cathode material; sodium cobalt oxide; structure change; charge compensation mechanism; structure-activity relationship

\section{1 引言}

日益增长的清洁能源需求促使着电池研究不断向
更高容量、更高能量密度和更低成本 ${ }^{[1-2]}$ 的方向实现突 破. 近些年来, 与锂离子电池工作原理类似的钠离子电 池凭借分布广泛、资源丰富的钠资源受到广泛关注，有

* E-mail: yyang@xmu.edu.cn; Tel.: 0592-2185753

Received June 9, 2021; published July 19, 2021.

Project supported by the National Key Research and Development Program of China (No. 2016YFB0901502) and funding project of the Top-notch Students Scientific Development Pilot Program (Chemistry) of Xiamen University.

国家重点研发专项课题(No. 2016YFB0901502)和厦门大学化学学科拔尖学生培养试验计划资助项目. 
望成为锂离子电池在大规模储能领域的良好替代品 ${ }^{[3]}$. 为了进一步实现钠离子电池的实际应用, 发展兼具高容 量和良好循环稳定性的电极材料十分重要. 在基础研究 过程中, 研究者需要深入分析钠离子电池电极材料的失 效机理并建立相应构效关系, 为进一步研发新型电极材 料提供理论依据. 随着电化学储能领域的飞速发展, 目 前钠离子电池的电极材料机理和性能的研究取得了较 大的进展, 但要想进一步在原子和离子尺度来理解材料 的构效关系, 一方面亟需提高现有表征技术的时空尺度 分辨能力以及对数据的解析能力, 另一方面需要选取合 适的典型结构来对材料的失效机制进行细致的分析.

无论是在锂离子电池还是在钠离子电池中, 正极材 料都是影响电池性能和成本的关键组成部分. 基于现有 理解层次, 研究者们针对不同结构类型的材料展开了研 究. 其中层状过渡金属氧化物 $\left(\mathrm{Li}_{x} \mathrm{TMO}_{2} / \mathrm{Na}_{x} \mathrm{TMO}_{2}, \mathrm{TM}\right.$ $=$ 过渡金属, 如 $\mathrm{Mn}, \mathrm{Ni}, \mathrm{Co}$ 等)凭借其比容量、优异的循 环稳定性等优势得到了深入研究, 如锂离子电池中的钴

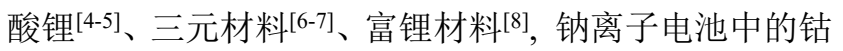

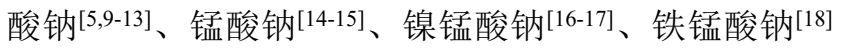
等系列材料. 其中相较于锂基材料, 具有电化学活性的 钠离子电池层状过渡金属氧化物种类更加丰富: 由从 $\mathrm{Ti}$ 到 $\mathrm{Ni}$ 的 7 种过渡金属 $\left(\mathrm{Ti}^{[19-20]}, \mathrm{V}^{[21]}, \mathrm{Cr}^{[22]}, \mathrm{Mn}^{[23-24]}, \mathrm{Fe}^{[25]}\right.$, $\mathrm{Co}^{[5,10-11,20]}, \mathrm{Ni}^{[20,26]}$ 组成的层状 $\mathrm{Na}_{x} \mathrm{MO}_{2}$ 都具有高活性, 可供 $\mathrm{Na}^{+}$进行脱嵌. 本课题组也针对 $\mathrm{Na}_{0.67} \mathrm{MnO}_{2}$ 和

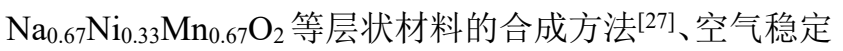

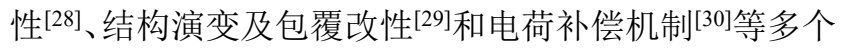
方面开展了工作. 表 1 对比了常见钠离子电池层状过渡 金属氧化物正极材料的特点.

表 1 常见钠离子电池层状过渡金属氧化物正极材料对比

Table 1 Comparison of common layered transition metal oxide cathode materials in sodium ion batteries

\begin{tabular}{|c|c|c|}
\hline 名称 ${ }^{a}$ & 优点 & 缺点 \\
\hline $\mathrm{NaMnO}_{2}$ & $\begin{array}{l}\text { Mn 自然丰度高, } \\
\text { 理论容量高( } 243 \mathrm{mAh} / \mathrm{g})\end{array}$ & $\begin{array}{l}\text { 存在 Jahn-Teller 畸变, } \\
\text { 充放电阶段多阶梯状曲线, } \\
\text { 结构稳定性差, 循环性能差 }\end{array}$ \\
\hline $\mathrm{NaFeO}_{2}$ & $\begin{array}{l}\text { Fe 自然丰度极高, } \\
\text { 低电压下充放电平台稳 } \\
\text { 定, } \\
\text { 电化学可逆性好 }\end{array}$ & $\begin{array}{l}\text { 质量比容量低, 高电位下存在不 } \\
\text { 可逆相变, 循环稳定性差 }\end{array}$ \\
\hline $\mathrm{NaCoO}_{2}$ & $\begin{array}{l}\text { 低电压下电化学可逆性 } \\
\text { 较好, 离子电导率高 }\end{array}$ & $\begin{array}{l}\text { Co 自然丰度极低, 成本高, } \\
\text { 容量低, 充放电曲线多平台, } \\
\text { 倍率性能差, 高电位下循环性能 } \\
\text { 差 }\end{array}$ \\
\hline
\end{tabular}

在多种钠离子电池层状过渡金属氧化物中, 钴酸钠 很早就受到了研究者们的关注. 早在 20 世纪 80 年代 ${ }^{[31]}$, 当钴酸锂首次被报道应用于锂离子电池正极材料 时 ${ }^{[32-33]}$, 钴酸钠作为正极材料在钠离子电池中的电化学 活性就已得到证实 ${ }^{[5,10-11,13]}$. 研究表明, 与钴酸锂相比, 钴酸钠的倍率性能和循环性能较差 ${ }^{[12,34]}$, 且钴资源在自 然界中的存储愈发有限、分布不均, 这些都阻碍了钴酸 钠的实际应用. 图 1 展示了钴酸钠研究发展历史和重要
研究进展, 可以看到从 1981 年 Delmas 等 ${ }^{[10]}$ 最初报道钴 酸钠的储钠性能至今 40 年间的发展历程中, 钴酸钠材 料逐渐由初期的储能应用为导向的研究, 转变为 21 世 纪初的热电、超导等新应用方向基础研究. 随着近年来 钠离子电池研究热点的重新回归, 钴酸钠也重新获得了 人们的关注, 但更多的研究趋势是集中在针对其失效机 制的解释与构效关系的探寻上. 可见, 从钴酸钠自身电 化学特点出发, 基于现有先进表征技术针对钴酸钠进行 电化学过程的机理分析在如今仍具有重要意义: 一方面 针对其充放电过程中丰富的相变进行研究可以促进固 态电化学的发展; 另一方面针对其 $\mathrm{Na}^{+}$-空位有序排布 和电荷补偿机理进行研究也能够为层状过渡金属氧化 物失效机理的研究提供新的思路. 因此, 基于钴酸钠材 料的常见结构, 本文综述了近年来钴酸钠作为钠离子电 池正极材料在充放电过程中的结构演变及电荷补偿机 制的研究进展, 讨论了上述机理对化学性能的影响.

\section{2 钴酸钠合成相图、晶体结构与电化学性能}

\section{1 钴酸钠的合成相图与常见晶体结构}

钴酸钠是由 $\mathrm{Na}$ 层和 $\mathrm{CoO}_{2}$ 层交替堆叠组成的. 其中 $\mathrm{Na}^{+}$在电化学过程中能进行脱嵌, 且迁移速率快. 与其 他层状过渡金属氧化物材料类似, 钴酸钠的晶体结构也 依赖于 $\mathrm{Na} / \mathrm{Co}$ 比例. Delmas 等 ${ }^{[41]}$ 在 1980 年对这些结构 进行了分类, 即 $\mathrm{P} 2, \mathrm{P} 3$ 和 $\mathrm{O} 2, \mathrm{O} 3$ 型等结构, 其中 $\mathrm{O} / \mathrm{P}$ 指 $\mathrm{Na}^{+}$与氧离子为八面体形配位/三棱柱形配位, 数字表示 $\mathrm{CoO}_{2}$ 层垂直于钠离子层堆叠的重复周期数. 目前已有 报道的层状 $\mathrm{Na}_{x} \mathrm{CoO}_{2}(0.5<x<1)$ 材料晶体结构主要包 括: $\mathrm{P} 2^{[10,12,36-37]} 、 \mathrm{P}^{\prime} 3^{[10,37,42]} 、 \mathrm{P} 3^{[12,37,42]} 、 \mathrm{O}^{\prime} 3^{[10,12,37]}$ 、 $\mathrm{O} 3^{[10,12,37]}$ 类型, 其中符号 “,”表示结构存在畸变.

\section{1 .1 合成相图}

1973 年, Fouassier 等报道了 $\mathrm{Na}_{x} \mathrm{CoO}_{2}$ 的合成相图, 并指出通过高温固相反应合成可得到 $\mathrm{Na}_{x} \mathrm{CoO}_{2}$ 的四种 结构, 即 $\alpha(\mathrm{O} 3), \alpha^{\prime}\left(\mathrm{O}^{\prime} 3\right), \beta\left(\mathrm{P}^{\prime} 3\right)$ 和 $\gamma(\mathrm{P} 2) \mathrm{Na}_{x} \mathrm{CoO}_{2} .2014$ 年 Ceder 等 ${ }^{[37]}$ 进一步构建了 $\mathrm{Na}_{x} \mathrm{CoO}_{2}$ 在空气中的合成相 图 $\left(450 \sim 750{ }^{\circ} \mathrm{C}, n(\mathrm{Na}): n(\mathrm{Co})=0.60 \sim 1.05\right)$, 如图 2a, 观察到 $\mathrm{O} 3, \mathrm{O}^{\prime} 3, \mathrm{P}^{\prime} 3$ 和 $\mathrm{P} 2$ 型 $\mathrm{Na}_{x} \mathrm{CoO}_{2}$ 晶体结构的单相稳 定区域; 同时还发现钴酸钠材料最终的化学组成仅由系 统热力学驱动, 例如初始投料范围较大时, $\mathrm{O} 3, \mathrm{O}^{\prime} 3$ 和 $\mathrm{P}^{\prime} 3$ 的纯相最终只能分别以 $x=1.00 、 0.83$ 和 0.67 的固定 化学计量比形成, $\mathrm{P} 2$ 相纯相范围稍大, $\mathrm{Na}^{+}$含量范围 $x$ 可 以从 0.68 变化到 0.76 .

\section{1 .2 晶体结构}

$\mathrm{O} 3-\mathrm{Na}_{x} \mathrm{CoO}_{2}(0.77 \leqslant x \leqslant 1)$ 为典型的 $\alpha-\mathrm{NaFeO}_{2}$ 结构, 其结构中钠离子和钴离子都是通过与氧以八面体形式 进行配位, 氧原子的堆积方式为 $\mathrm{ABCABC}$, 空间群为 $R \overline{3} m$, 如图 $2 \mathrm{~b}$ 所示. 相较于 $\mathrm{O} 3$ 相, $\mathrm{O}^{\prime} 3$ 相在结构中出现 畸变, 形成了对称性较低的单斜结构, 其空间群为 
1988, Shacklette:

The first NIBs were demonstrated

> Cathode: P2 \& P3- $\mathrm{Na}_{x} \mathrm{CoO}_{2}$

> Anode: PPP and $\mathrm{Na}_{x} \mathrm{~Pb}$

2003, Takada: $\mathrm{Na}_{x} \mathrm{CoO}_{2} \cdot y \mathrm{H}_{2} \mathrm{O}(x<0.35, y<1.3)$

$>$ Superconductivity

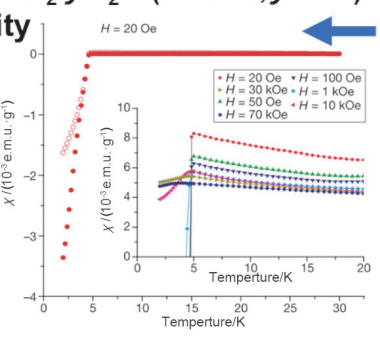

2004, Zandbergen: Electron Diffraction $>$ Sodium ion ordering

2011, Delmas: P2- $\mathrm{Na}_{x} \mathrm{CoO}_{2}$--in situ XRD

\section{$>$ Phase changes}

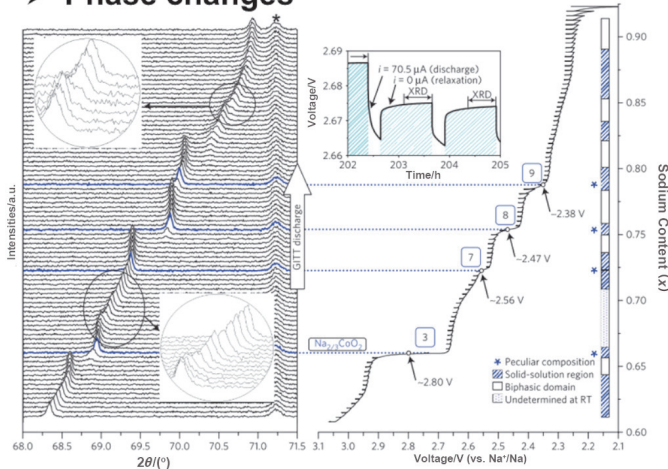

2014, Ceder: O3- $\mathrm{Na}_{x} \mathrm{CoO}_{2}$--in situ XRD

$>$ Phase changes

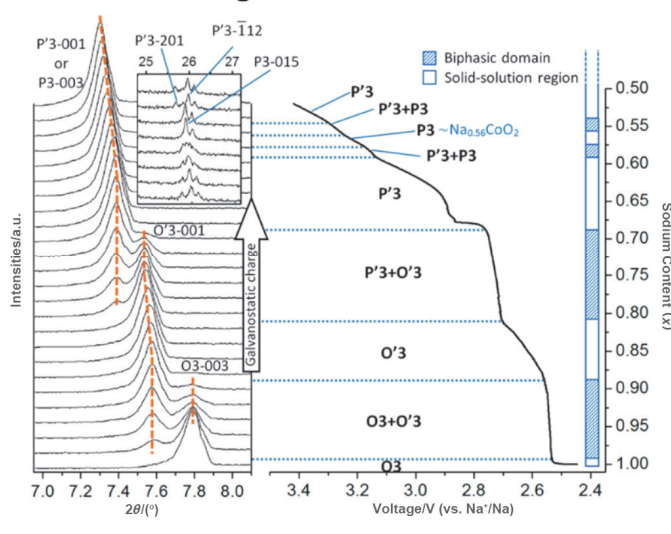

1981, Delmas:

Four types of $\mathrm{Na}_{x} \mathrm{CoO}_{2}$

$>0.55 \leqslant x \leqslant 0.60\left(\mathrm{P}^{\prime} 3\right), 0.64 \leqslant x \leqslant 0.74(\mathrm{P} 2)$

> $x=0.77\left(0^{\prime} 3\right), x=1(03)$

1997, Terasaki: $\mathrm{Na}_{0.5} \mathrm{CoO}_{2}$

--thermoelectric material

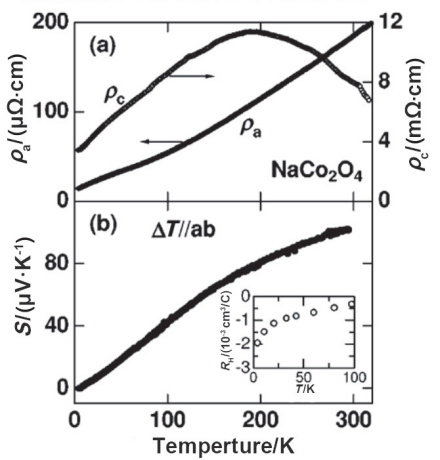

2013, Fu: P2-Na $\mathrm{CoO}_{2}-$-XPS

$>$ Co valence change

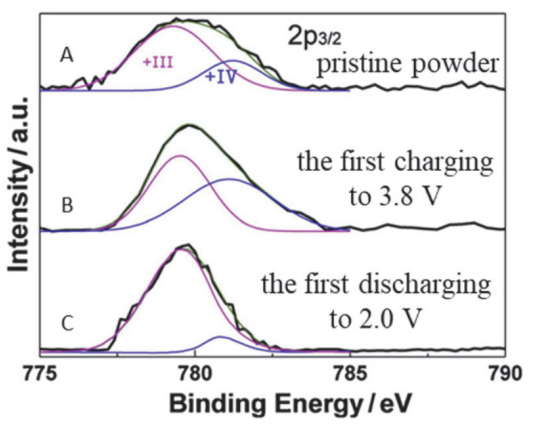

2021, Hausbrand: $\mathrm{Na}_{x} \mathrm{CoO}_{2}-$-XANES

- Anionic redox
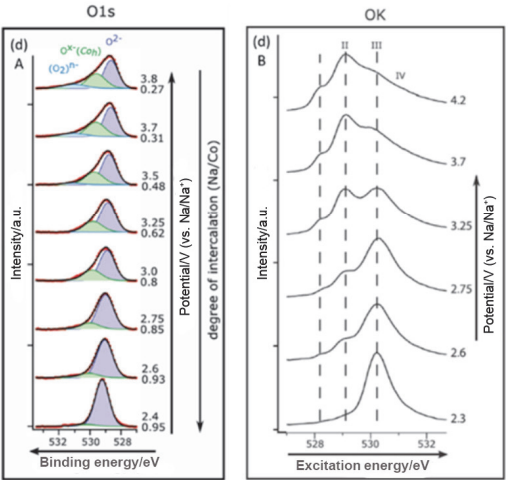

图 1 1981 2021 年钴酸钠研究发展历史和重要研究进展 ${ }^{[35-40]}$

Figure 1 The development history and important research progress of sodium cobalt oxide from 1981 to $2021^{[35-40]}$

Reproduced with permission ${ }^{[35]}$, Copyright 2003 Nature Publishing group. Reproduced with permission ${ }^{[36]}$, Copyright 2011 Nature Publishing group. Reproduced with permission ${ }^{[37]}$, Copyright 2014 American Chemical Society. Reproduced with permission ${ }^{[38]}$, Copyright 1997 American physical Society. Reproduced with permission ${ }^{[39]}$, Copyright 2013 Elsevier. Reproduced with permission ${ }^{[40]}$, Copyright 2021 Elsevier.

$C 2 / m$ (图 2c).

$\mathrm{P} 3-\mathrm{Na}_{x} \mathrm{CoO}_{2}(0.55 \leqslant x \leqslant 0.60)$ 的结构中钴离子与氧 都是通过以八面体形式进行配位, 而钠离子和氧以三棱 柱形式进行配位, 其结构中所有的三棱柱位点均与一个 八面体共面, 与另外三个八面体共边, 氧堆积方式为
$\mathrm{ABBCCA}$, 空间群为 $R \overline{3} m$, 如图 $2 \mathrm{~d}$ 所示. 而 $\mathrm{P}^{\prime} 3$ 相与 $\mathrm{P} 3$ 相相比, $\mathrm{P}^{\prime} 3$ 相具有单斜畸变, 其空间群为 $C 2 / m$, 如图 $2 \mathrm{e}$ 所示.

$\mathrm{P} 2-\mathrm{Na}_{x} \mathrm{CoO}_{2}(0.64 \leqslant x \leqslant 0.74)$ 结构中所有的钴离子 与氧均以八面体形式配位, 而其中的钠离子和氧以三棱 


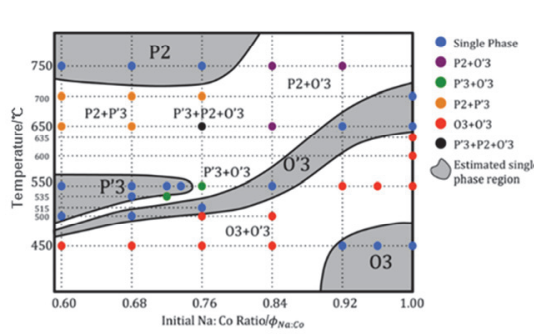

(a)

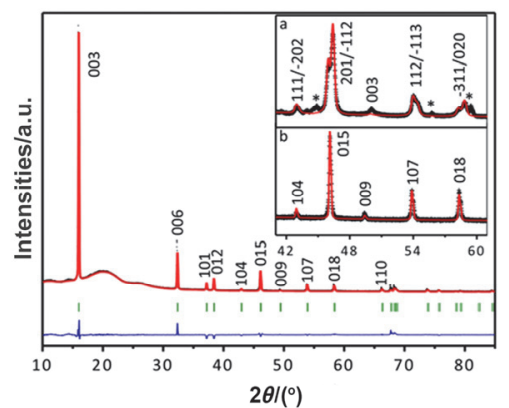

(d) $\mathrm{P} 3-\mathrm{Na}_{0.56} \mathrm{CoO}_{2}$

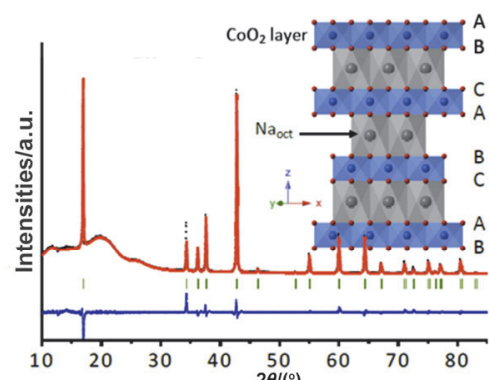

(b) $\mathrm{O} 3-\mathrm{Na}_{1.00}^{2 \theta(())} \mathrm{CoO}_{2}$

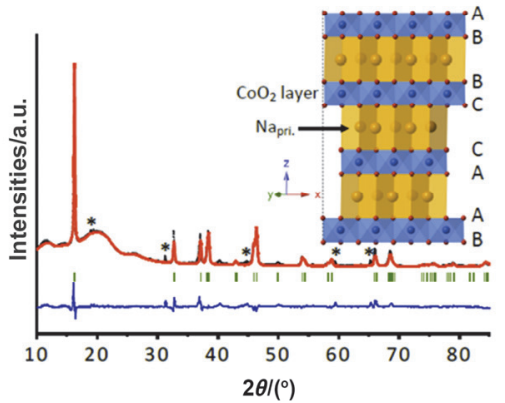

(e) $\mathrm{P}^{\prime} 3-\mathrm{Na}_{0.67} \mathrm{CoO}_{2}$

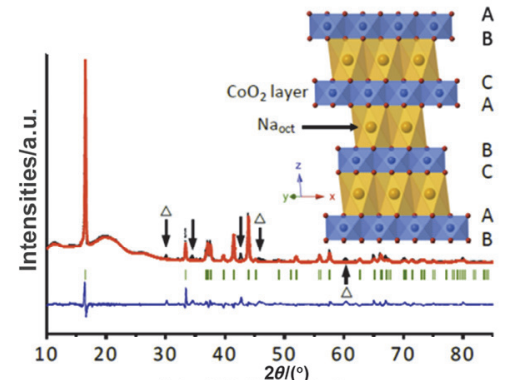

(c) $\mathrm{O}^{\prime} 3-\mathrm{Na}_{083}^{2 \theta 1 / 9} \mathrm{COO}_{2}$

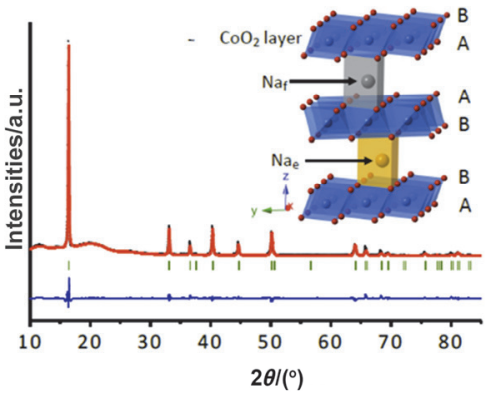

(f) $\mathrm{P} 2-\mathrm{Na}_{0.76} \mathrm{CoO}_{2}$

图 $2 \mathrm{Na}_{x} \mathrm{CoO}_{2}$ 的(a)合成相图 ${ }^{[37]}$ 和 $(\mathrm{b} \sim \mathrm{f}) \mathrm{X}$ 射线衍射 $(\mathrm{XRD})$ 谱图 ${ }^{[37]}$

Figure 2 (a) Synthesis phase diagram and (b-f) XRD patterns of $\mathrm{Na}_{x} \mathrm{CoO}_{2}{ }^{[37]}$

Reproduced with permission ${ }^{[37]}$, Copyright 2014 American Chemical Society

柱形式配位, 并且结构中的钠离子含有两种不同类型的 位点, 记为 $\mathrm{Na} 1, \mathrm{Na} 2$, 其中 $\mathrm{Na} 1$ 位点与八面体 $\left(\mathrm{MO}_{6}\right)$ 处 于共面状态, $\mathrm{Na} 2$ 位点与八面体 $\left(\mathrm{MO}_{6}\right)$ 处于共边状态, 结 构中氧为 $\mathrm{ABBA}$ 堆积, 空间群为 $P 6_{3} / m m c$, 如图 $2 \mathrm{f}$ 所示.

\section{2 钴酸钠的电化学性能研究}

目前已有大量文献研究报道了 $\mathrm{O} 3$ 型、 $\mathrm{P}^{\prime} 3$ 型和 $\mathrm{P} 2$ 型 $\mathrm{Na}_{x} \mathrm{CoO}_{2}$ 作为钠离子电池正极材料的电化学性能. 如 图 3a 所示, 在 $2.0 \sim 4.0 \mathrm{~V}$ 电压区间内, $\mathrm{O} 3, \mathrm{P}^{\prime} 3$ 和 $\mathrm{P} 2$ 型 $\mathrm{Na}_{x} \mathrm{CoO}_{2}$ 的首圈放电比容量分别为 140,130 和 120 $\mathrm{mAh} / \mathrm{g}$. 尽管三种晶型材料的结构存在明显区别, $\mathrm{Na}_{x} \mathrm{CoO}_{2}$ 在钠含量较低区域的充放电曲线形状基本相 同: 当钠含量 $x<0.7$ 时, 对应 $\mathrm{Na}_{x} \mathrm{CoO}_{2}$ 的电压平台都几 乎在相似区域出现(平台范围依次为: $2.6 \sim 2.7 \mathrm{~V}, 2.8 \sim$ $2.9 \mathrm{~V}, 3.1 \sim 3.2 \mathrm{~V}, 3.6 \sim 3.7 \mathrm{~V}, 3.8 \sim 3.9 \mathrm{~V}) . \mathrm{P} 2-\mathrm{Na}_{x} \mathrm{CoO}_{2}$ 在 $2.6 \mathrm{~V}$ 以下的充放电曲线与其他晶型有所区别, 出现了 多个阶梯状平台.

随着 $\mathrm{Na}^{+}$的脱嵌, 上述材料均伴随着较大体积变化. 在没有钠离子的情况下, 氧离子层将直接相对而处于不 稳定状态, 因此 $\mathrm{Na}^{+}$若发生过度脱出, 将导致晶体结构 发生不可逆变化. 由此可知, $\mathrm{Na}^{+}$脱嵌的范围应限制在 晶体结构不会发生剧烈改变的条件下, 但这将导致 $\mathrm{Na}_{x} \mathrm{CoO}_{2}$ 的实际比容量远低于理论比容量 $(235 \mathrm{mAh} / \mathrm{g})$, 阻碍其在钠离子电池中的应用 ${ }^{[39,43]}$. 尽管如此, 从另一 方面看, 该限制也为提升正极材料性能提供了指导方 向: 在充放电循环过程中增大 $\mathrm{Na}^{+}$的可逆利用对电池性 能的改善将非常有利. 目前已有研究通过元素掺杂/取
代的策略来提升 $\mathrm{Na}^{+}$的可逆利用率, 提高钠离子电池性 能 ${ }^{[4-47]} .2018$ 年 Mun 等 ${ }^{[4]}$ 设计了 $\mathrm{P} 2-\mathrm{Na}_{0.67} \mathrm{Co}_{1-x} \mathrm{Ti}_{x} \mathrm{O}_{2}$ $(x<0.2)$ 正极材料, 其在 $2 \sim 4.4 \mathrm{~V}$ 的范围下工作时具有 $166 \mathrm{mAh} / \mathrm{g}$ 的首圈放电比容量, 其中 $\mathrm{Na}_{0.67} \mathrm{Co}_{0.90} \mathrm{Ti}_{0.10} \mathrm{O}_{2}$ 在 100 次循环后仍具有较好的容量保持能力 $(115$ $\mathrm{mAh} / \mathrm{g}$ ), 而相同电压范围下 $\mathrm{Na}_{0.67} \mathrm{CoO}_{2}$ 的放电比容量已 经低于 $10 \mathrm{mAh} / \mathrm{g}$. 尽管如此, 目前针对钴酸钠材料的改 性研究并不充分, 钴酸钠本征性能上的劣势使得该材料 的改性研究关注度不高. 然而, 借鉴其他钠离子层状正 极材料的改性工作经验，或许能为解决钴酸钠材料性能 劣势提供指导性意见: 在传统设计思路上, 除了进行阳 离子掺杂，还可以通过表面包覆碳、氧化物等化合物手 段来增强材料在电化学过程中的结构稳定性; 同时目前 也有报道通过设计合成 $\mathrm{P} 2 / \mathrm{O} 3$ 堆积的复合结构材料来增 强层状氧化物材料的比容量和循环稳定性. 另一方面, 在钴酸钠本征性能存在劣势的背景之下，其较差性能背 后蕴含的丰富的失效机理信息, 也吸引着研究人员进一 步对其进行深入的失效机制研究.

与锂离子电池中常见的层状 $\mathrm{LiCoO}_{2}^{[49-51]}$ 相比(如图 $3 b$ 所示), $\mathrm{Na}_{x} \mathrm{CoO}_{2}$ 在充放电曲线上表现出了多个电压平 台, 表明随着 $\mathrm{Na}^{+}$的嵌入和脱出, $\mathrm{Na}_{x} \mathrm{CoO}_{2}$ 经历了非常复 杂的相变过程 ${ }^{[2]}$, 可能是层内 $\mathrm{Na}^{+} /$空位复杂的有序情 况所导致的 ${ }^{[53]}$. 这种 $\mathrm{Na}^{+} /$空位有序性现象可能是晶体 中多种离子间的相互作用进行能量最小化的结果，但目 前对该现象还没有直接可靠的表征研究. 结构决定性 质, 研究 $\mathrm{Na}_{x} \mathrm{CoO}_{2}$ 充放电过程中的多种相变机理, 了解 
$\mathrm{Na}_{x} \mathrm{CoO}_{2}$ 中 $\mathrm{Na}^{+}$/空位的有序性情况, 这对从 $\mathrm{Na}_{x} \mathrm{CoO}_{2}$ 出 发建立钠离子层状氧化物的构效关系具有指导意义.

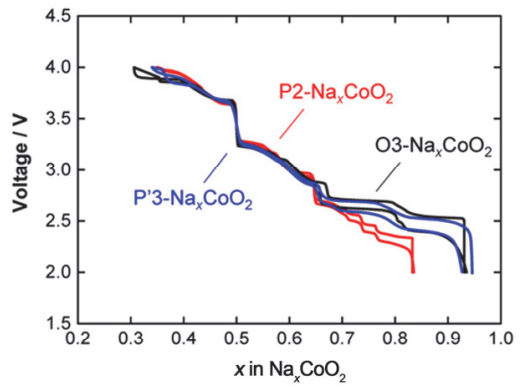

(a)

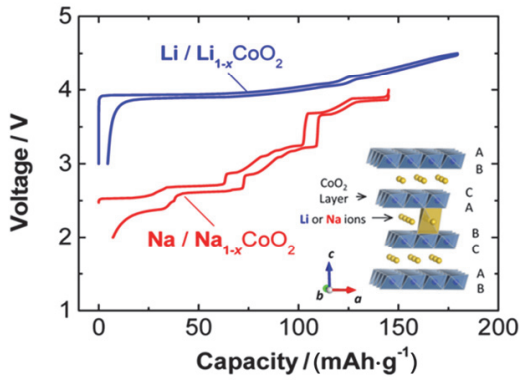

(b)

图 3 (a) $\mathrm{O} 3$ 型、 $\mathrm{P}^{\prime} 3$ 型和 $\mathrm{P} 2$ 型 $\mathrm{Na}_{x} \mathrm{CoO}_{2}$ 的充放电曲线比较; (b) $\mathrm{LiCoO}_{2}$ 和 $\mathrm{NaCoO}_{2}$ 的充放电曲线比较 ${ }^{[10,49]}$

Figure 3 (a) Comparison of charge/discharge curves of O3-, P'3-, and P2-type $\mathrm{Na}_{x} \mathrm{CoO}_{2}$ cells; (b) comparison of charge/discharge curves of $\mathrm{LiCoO}_{2}$ and $\mathrm{NaCoO}_{2}$ cells $s^{[10,49]}$

Reproduced with permission ${ }^{[49]}$, Copyright 2014 American Chemical Society.

\section{3 钴酸钠结构演变研究进展}

\section{1 钴酸钠相变研究}

\subsubsection{O3-O'3-P'3-P3-P'3 型相变}

由于 $\mathrm{O} 3$ 和 $\mathrm{P}^{\prime} 3$ 仅在 $\mathrm{Na}^{+}$含量和 $\mathrm{Na}-\mathrm{O}$ 配位方式上有 所不同, 可推测这些相在充放电过程中将通过层间滑移 引起相变, 进而发生晶型上的相互转化 ${ }^{[54-55]}$. 在充放电 曲线中, 双相的出现通常会导致电压平台的出现, 而单 相阶段则与曲线中的电压阶跃升降有关, 固溶体与倾斜 曲线相对应. Delmas 等 ${ }^{[10]}$ 在 1981 年就指出 O3 型 $\mathrm{Na}_{x} \mathrm{CoO}_{2}$ 在充放电过程中会发生 $\mathrm{O} 3-\mathrm{O}^{\prime} 3-\mathrm{P}^{\prime} 3$ 的可逆相转 变(如图 4a). 1988 年 Shacklette 等 ${ }^{[12]}$ 结合 $-\mathrm{d} E / \mathrm{d} x$ 曲线与非 原位 XRD 进一步证明了在充放电过程中 $\mathrm{P}^{\prime} 3-\mathrm{Na}_{x} \mathrm{CoO}_{2}$ 将发生 O3-O'3-P'3 的相转变(图 4b), $x=1$ 时的 $\mathrm{NaCoO}_{2}$ 对应于 $\mathrm{O} 3$ 相, 在 $x=0.85$ 附近出现的一个峰对应于 $\mathrm{O}^{\prime} 3$ 相, 而在 $x=0.7$ 附近出现的较高峰对应于 $\mathrm{P}^{\prime} 3$ 相.

原位 XRD 是表征充放电过程中材料相变的强有力 手段. 2014 年 Ceder 等[37]结合原位 XRD 监测了 O3 型 $\mathrm{Na}_{x} \mathrm{CoO}_{2}$ 充电过程中的相变情况 (如图 4c 所示). 在材料 充电至 2.4 3.4 V 时观察到 $\mathrm{O} 3, \mathrm{O}^{\prime} 3$ 和 $\mathrm{P}^{\prime} 3$ 型的单、双 相区域. 此外, 在充电末期(3.15 3.4 V)观察到(201)和 (112)峰同时出现, 并且(015)峰发生连续分裂(图 4c 内插 图), 归属于 $\mathrm{P}^{\prime} 3-\mathrm{P} 3-\mathrm{P}^{\prime} 3$ 相变, 这说明在早期报道的
O3-O'3-P'3 相变的基础上, P'3 相还将发生 P'3-P3-P'3 的 转变. 为了深入认识 P'3-P3-P'3 相变, 2008 年 Blangero 等 ${ }^{[42]}$ 曾通过 ${ }^{23} \mathrm{Na}$ 魔角旋转核磁共振 $\left({ }^{23} \mathrm{Na}\right.$ MAS-ssNMR) 观察 $\mathrm{P}^{\prime} 3-\mathrm{Na}_{0.62} \mathrm{CoO}_{2}$ 发生的 $\mathrm{P}^{\prime} 3-\mathrm{P} 3$ 高温相变(图 4d). $\mathrm{P}^{\prime} 3-\mathrm{Na}_{0.62} \mathrm{CoO}_{2}$ 的 ${ }^{23} \mathrm{Na}$ 化学位移信号峰形状具有二阶相 互作用的特征, 这是 $\mathrm{Na}^{+}$处于非对称环境中的典型特征, 而该特征在升温时产生的 $\mathrm{P} 3-\mathrm{Na}_{0.62} \mathrm{CoO}_{2}$ 核磁信号中消 失. 通过 XRD 所得材料 $\mathrm{Na}^{+}$配位环境示意图(图 4e)进 一步表明, $\mathrm{P}^{\prime} 3-\mathrm{Na}_{0.62} \mathrm{CoO}_{2}$ 相较于 $\mathrm{P} 3-\mathrm{Na}_{0.62} \mathrm{CoO}_{2}, \mathrm{Na}^{+}$偏 离了其所在的六棱柱配位中心, 这可能对应于一种 $\mathrm{Na}^{+}$ /空位的无序化表现, P'3 相可能是 P3 相在 $\mathrm{Na}^{+} /$空位排序 上产生的超结构.

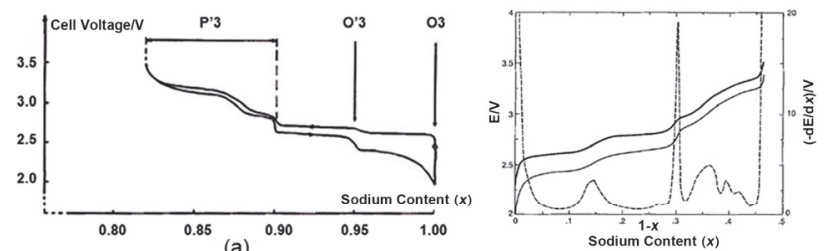

(b)

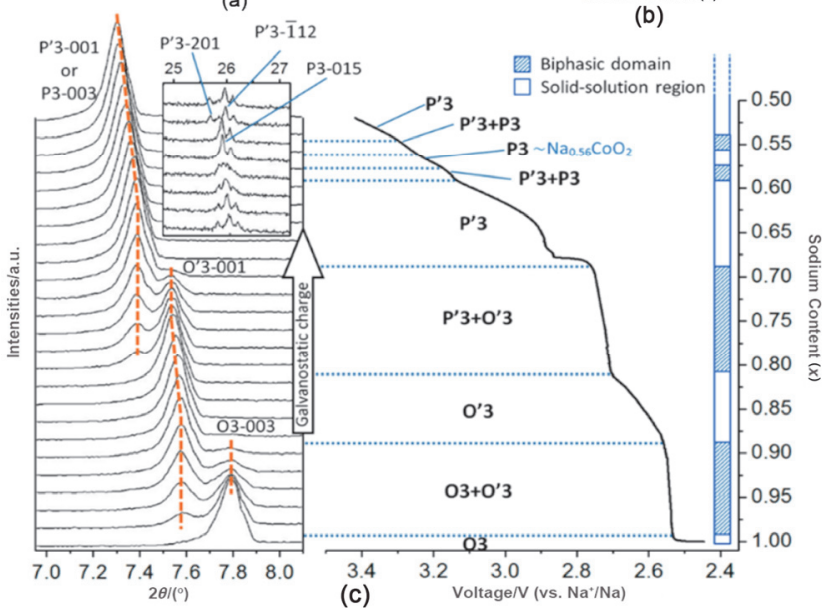

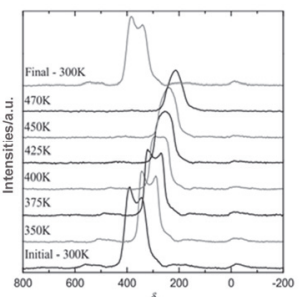

(d)

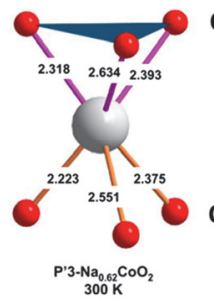

(e)

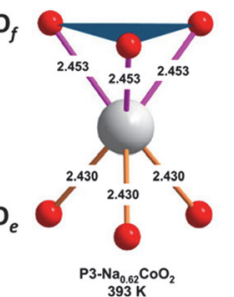

$\mathrm{P}_{393 \mathrm{~K}} \mathrm{Na}_{0.62} \mathrm{CoO}_{2}$
图 4 (a) $\mathrm{O} 3-\mathrm{Na}_{x} \mathrm{CoO}_{2}$ 的充放电相变情况 ${ }^{[10]}$; (b) $\mathrm{P}^{\prime} 3-\mathrm{Na}_{x} \mathrm{CoO}_{2}$ 的充电 (左)与 $-\mathrm{d} E / \mathrm{d} x$ 曲线 (右 $)^{[11]}$; (c) $\mathrm{O} 3-\mathrm{Na}_{x} \mathrm{CoO}_{2}$ 的充电曲线和原位 XRD 表征 结果 ${ }^{[37]}$; (d) 不同温度下 $\mathrm{P}^{\prime} 3-\mathrm{Na}_{x} \mathrm{CoO}_{2}$ 的 ${ }^{23} \mathrm{Na}$ MAS-NMR 谱 ${ }^{[42]}$; (e) $\mathrm{P}^{\prime} 3$ 和 P3- $\mathrm{Na}_{x} \mathrm{CoO}_{2}$ 的 $\mathrm{Na}^{+}$配位环境示意图 ${ }^{[42]}$

Figure 4 (a) Phase change in the charge-discharge process of $\mathrm{O} 3-\mathrm{Na}_{x} \mathrm{CoO}_{2}{ }^{[10]}$; (b) charge curve to the left and $-\mathrm{d} E / \mathrm{d} x$ curve to the right for $\mathrm{P}^{\prime} 3-\mathrm{Na}_{x} \mathrm{CoO}_{2}{ }^{[11]}$; (c) charge curve and in situ XRD of O3- $\mathrm{Na}_{x} \mathrm{CoO}_{2}{ }^{[37]}$; (d) ${ }^{23} \mathrm{Na}$ MAS-NMR of $\mathrm{P}^{\prime} 3-\mathrm{Na}_{x} \mathrm{CoO}_{2}$ in different temperature ${ }^{[42]}$; (e) $\mathrm{NaO}_{6}$ prismatic environments for both $\mathrm{P}^{\prime} 3$ and $\mathrm{P} 3-\mathrm{Na}_{x} \mathrm{CoO}_{2}$ to the right ${ }^{[42]}$

Reproduced with permission ${ }^{[10]}$, Copyright 1981 Elsevier. Reproduced with permission ${ }^{[11]}$, Copyright 1983 Elsevier. Reproduced with permission $^{[37]}$, Copyright 2014 American Chemical Society. Reproduced with permission $^{[42]}$, Copyright 2008 the American physical Society.

层状 $\mathrm{LiCoO}_{2}$ 同样也为 $\mathrm{O} 3$ 型结构, 与图 5 对比了 $\mathrm{O} 3-\mathrm{Na}_{x} \mathrm{CoO}_{2}$ 与 $\mathrm{O} 3-\mathrm{Li}_{x} \mathrm{CoO}_{2}$ 在充放电过程中的相变情况. 
相比于 $\mathrm{O} 3-\mathrm{Li}_{x} \mathrm{CoO}_{2}, \mathrm{O} 3-\mathrm{Na}_{x} \mathrm{CoO}_{2}$ 所能保持结构的 $x\left(\mathrm{Li}^{+} /\right.$ $\mathrm{Na}^{+}$) 含量范围更窄, 即几乎当 $\mathrm{NaCoO}_{2}(x=1)$ 开始充电 时, 六方 $\mathrm{O} 3$ 相就开始转变为单斜 $\mathrm{O}^{\prime} 3$ 相. 而当 $x=0.5$ 时, $\mathrm{O} 3-\mathrm{Li}_{x} \mathrm{CoO}_{2}$ 才开始转变为单斜相. 晶相的转变往往 伴随着剧烈的体积变化: $\mathrm{O} 3-\mathrm{Na}_{x} \mathrm{CoO}_{2}$ 转化为单斜相后 $c$ 轴膨胀了 $3.1 \%$; 而 $\mathrm{O} 3-\mathrm{Li}_{x} \mathrm{CoO}_{2}$ 转化为单斜相后 $c$ 轴膨 胀了 $2.3 \%$. 通过上述可以发现, 相较于 $\mathrm{O} 3-\mathrm{Li}_{x} \mathrm{CoO}_{2}$, $\mathrm{O} 3-\mathrm{Na}_{x} \mathrm{CoO}_{2}$ 在充放电过程中发生的相变更多(这与其充 放电曲线的表现是一致的), 同时伴随着更加剧烈的体 积变化, 结构保持能力更差.

尽管如此, 就钴酸钠材料自身而言, 其在 O3 和 O'3 相中的 $\mathrm{Na}^{+}$需要通过四面体空位迁移到相邻 $\mathrm{Na}^{+}$八面体 位点, 而 $\mathrm{P} 3$ 和 $\mathrm{P}^{\prime} 3$ 相中的 $\mathrm{Na}^{+}$则可以通过层间平面直接 迁移到相邻的 $\mathrm{Na}^{+}$棱柱形位点, 这使得 $\mathrm{P}^{\prime} 3$ 和 $\mathrm{P} 3$ 相可以 在相对更大的 $\mathrm{Na}^{+}$含量范围内实现 $\mathrm{Na}^{+}$可逆的脱嵌和相 对更快的扩散, 从而使得可逆容量更大, 倍率性能更 好 ${ }^{[56]}$. 这可能说明 O3-O'3-P'3-P3-P'3 相变在一定范围内 有利于材料倍率性能的提升. 但相变本身伴随的离子迁 移将导致放电比容量下降, 电池极化增加, 相变过程中 引起的体积变化可能在晶格中产生内应力, 进而导致晶 格裂纹的产生 ${ }^{[57]}$, 对循环稳定性产生不利影响.

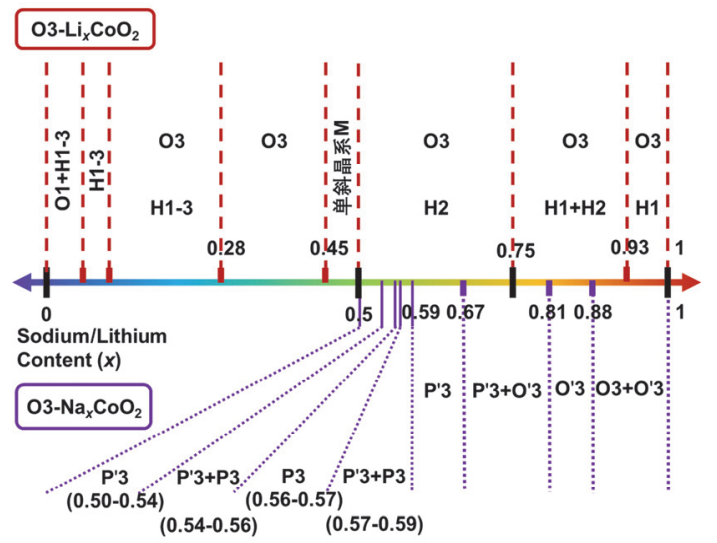

图 $5 \mathrm{O} 3-\mathrm{Li}_{x} \mathrm{CoO}_{2}$ 与 $\mathrm{O} 3-\mathrm{Na}_{x} \mathrm{CoO}_{2}$ 在充放电过程中的相变情况对比 Figure 5 Comparison of phase changes of $\mathrm{O} 3-\mathrm{Li}_{x} \mathrm{CoO}_{2}$ 与 $\mathrm{O} 3-\mathrm{Na}_{x} \mathrm{CoO}_{2}$ in the charging and discharging processes

\subsubsection{P2 型相变}

由上述可知, 从长程结构上看, $\mathrm{P} 2$ 型 $\mathrm{Na}_{x} \mathrm{CoO}_{2}$ 与 $\mathrm{P} 3$ 和 $\mathrm{O} 3$ 型的结构差距较大, 这种差距同样体现在局域结 构上. 2009 年 Carlier 等 ${ }^{[58]}$ 通过 ${ }^{23} \mathrm{Na}$ 魔角旋转核磁共振 (MAS-ssNMR) 对 P2 型和 P'3 型 $\mathrm{Na}_{x} \mathrm{CoO}_{2}$ 进行了表征, 发 现升高温度对 P2 和 $\mathrm{P}^{\prime} 3$ 型 $\mathrm{Na}_{x} \mathrm{CoO}_{2}$ 信号形状的改变完 全不同: 如图 6a 所示, P2 相中信号峰越来越尖锐而 P'3 相中信号峰变得模糊, 这说明两种堆叠方式中存在不同 的 $\mathrm{Na}^{+}$化学环境和 $\mathrm{Na}-\mathrm{Co}$ 相互作用. 长短程结构上的差 异将进一步反映到结构转化上: $\mathrm{P} 2-\mathrm{Na}_{x} \mathrm{CoO}_{2}$ 与 $\mathrm{O} 3$ 或 $\mathrm{P} 3$ 相的结构转化需要断裂 $\mathrm{Co}-\mathrm{O}$ 键, 该过程存在势垒阻 碍. 相较于其他两种晶型材料, $\mathrm{P} 2-\mathrm{Na}_{x} \mathrm{CoO}_{2}$ 可以在更大 的 $\mathrm{Na}^{+}$含量范围内保持结构. 2011 年 Delmas 等 ${ }^{[36]}$ 通过结
合恒电流间歇滴定技术(GITT) 及原位 XRD 表征了 $\mathrm{P} 2-\mathrm{Na}_{x} \mathrm{CoO}_{2}$ 在放电过程中的结构变化与电化学性能间 的关系, 如图 $6 \mathrm{~b}$ 所示, $\mathrm{P} 2-\mathrm{Na}_{x} \mathrm{CoO}_{2}$ 在 $2.0 \sim 3.8 \mathrm{~V}$ 范围内 具有约为 $125 \mathrm{mAh} / \mathrm{g}$ 的可逆容量, 这则与该晶型较好的 结构保持能力有关. 另外, 从原位 XRD 中可以看到(如 图 6c)(008)衍射峰在放电阶段发生了多次裂分，这直接 说明了 $\mathrm{P} 2-\mathrm{Na}_{x} \mathrm{CoO}_{2}$ 在放电过程中存在多个相变. Kim 等 ${ }^{[59]}$ 通过原位 XRD 在 $2.0 \sim 3.5 \mathrm{~V}$ 的充放电过程中进一 步观察到 $\mathrm{Na}_{x} \mathrm{CoO}_{2}$ 的 $c$ 轴和 $a$ 轴对应的衍射峰位置(如 (002), (004)和(100)) 随着 $\mathrm{Na}^{+}$脱嵌而移动(如图 6d), 这些 峰位移多以连续移动为主, 同时伴随着一些峰的裂分, 但首轮充放电过程未见相变和体积变化对材料电化学 性能有明显破坏，表明在 $2 \sim 3.5 \mathrm{~V}$ 范围内发生的相变和 体积变化是良性的, 这也是材料具有较高可逆容量的原 因之一。

总而言之, $\mathrm{P} 2$ 型 $\mathrm{Na}_{x} \mathrm{CoO}_{2}$ 具有较好的结构保持能力. 从离子扩散能力来看, 对于 $\mathrm{P} 2-\mathrm{Na}_{x} \mathrm{CoO}_{2}(0.54 \leqslant x \leqslant 0.85)$ 时, 材料平均 $\mathrm{Na}^{+}$扩散系数 $D_{\mathrm{Na}}^{+}$为 $4 \times 10^{-11} \sim 1.2 \times 10^{-10}$ $\mathrm{cm}^{2} / \mathrm{s}^{[60-62]}$, 相较于 $\mathrm{LiCoO}_{2}$ 中 $\mathrm{D}_{\mathrm{Li}}{ }^{+}$处于 $10^{-11} \sim 10^{-12} \mathrm{~cm}^{2} / \mathrm{s}$ 数量级, 表明 $\mathrm{P} 2-\mathrm{Na}_{x} \mathrm{CoO}_{2}$ 材料具有更好的倍率性能和 能量效率. 同时, 由于 $\mathrm{P} 2$ 相的结构保持能力在一定程度 上可以缓冲相变和体积变化带来的影响, 充放电过程中 发生的相变和体积变化对于电化学性能并未造成明显 恶性影响. 另外, 从充放电曲线和原位 XRD 中可以看 到存在较多相互接近的单相区域，这可能意味着不同 $\mathrm{Na}^{+}$含量下，材料结构中不同层间的 $\mathrm{Na}^{+} /$空位有序情况 可能不同, 这意味着若将不同层中的 $\mathrm{Na}^{+} /$空位有序进 行简单趋同描述，很大程度上可能并不能足以解释原位 技术表征中的现象.

\section{2 钴酸钠 $\mathrm{Na}^{+} /$空位有序性研究}

由于 $\mathrm{Na}_{x} \mathrm{CoO}_{2}$ 可通过改变 $\mathrm{Na}^{+}$含量调节 $\mathrm{Co}$ 价态, 得到了凝聚态物理界的广泛关注, 出现了一系列关于 $\mathrm{Na}_{x} \mathrm{CoO}_{2}$ 物理特性的研究, 随后 $\mathrm{Na}_{0.35} \mathrm{CoO}_{2} \bullet 1.3 \mathrm{H}_{2} \mathrm{O}$ 超导 性质的发现 ${ }^{[63]}$ 也进一步激发了人们对于 $\mathrm{Na}_{x} \mathrm{CoO}_{2}$ 材料 内部 $\mathrm{Na}^{+} /$空位特性的研究兴趣. 2004 年 Foo 等[64]报道了 $\mathrm{Na}_{x} \mathrm{CoO}_{2}(0.31<x<0.75)$ 的磁相图, 如图 7a 所示, 随着 $\mathrm{Na}^{+}$含量 $(x)$ 的增加, $\mathrm{Na}_{x} \mathrm{CoO}_{2}$ 从顺磁性金属转化为带荷 绝缘体, 然后变为 “Curie-Weiss” 金属, 最后转为弱磁 矩-磁有序状态。这些特殊性质与不同 $\mathrm{Na}^{+}$含量下的 $\mathrm{Na}^{+}$/空位耦合情况有关. 同时, $\mathrm{Na}_{x} \mathrm{CoO}_{2}$ 作为钠离子电 池正极材料时, 其电化学性能也受到 $\mathrm{Na}^{+} /$空位有序情 况的影响. $\mathrm{Na}^{+} /$空位有序情况是多个离子相互作用下能 量最小化的结果, 观察不同 $\mathrm{Na}^{+}$含量下的 $\mathrm{Na}^{+} /$空位的有 序情况, 对于进一步理解 $\mathrm{Na}_{x} \mathrm{CoO}_{2}$ 在热电范畴的特殊性 质和建立 $\mathrm{Na}_{x} \mathrm{CoO}_{2}$ 局域结构变化与电化学性能的构效 关系具有重要作用. 

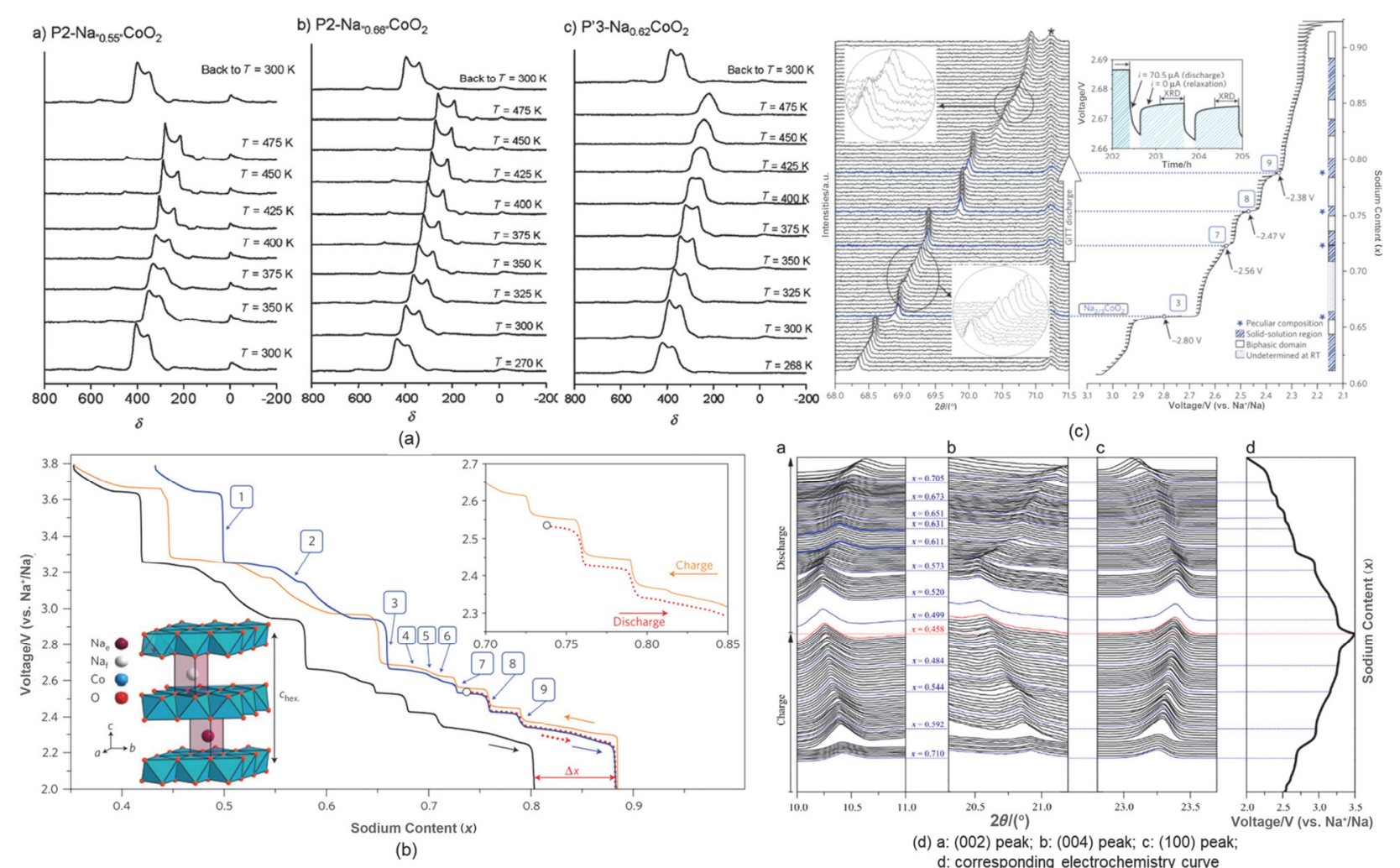

图 6 (a)三种 $\mathrm{Na}_{x} \mathrm{CoO}_{2}$ 材料随温度变化的 ${ }^{23} \mathrm{Na}$ MAS NMR 谱 ${ }^{[58]}$; (b) $\mathrm{Na}_{0.7} \mathrm{CoO}_{2}$ 的电化学循环及相变情况 ${ }^{[36]}$; (c) P2- $\mathrm{Na}_{x} \mathrm{CoO}_{2}$ 放电时的 $\mathrm{GITT}$ 及原位 XRD 表征情况 ${ }^{[36]} ;$ (d) $\mathrm{Na}_{x} \mathrm{CoO}_{2}$ 首轮充放电过程中的原位 XRD 变化 ${ }^{[59]}$

Figure 6 (a) ${ }^{23} \mathrm{Na}$ MAS NMR spectra recorded as a function of temperature for three materials ${ }^{[58]}$; (b) cycling and phase transitions of $\mathrm{Na}_{0.7} \mathrm{CoO}_{2}{ }^{[36]} ;$ (c) GITT and in situ XRD of $\mathrm{P} 2-\mathrm{Na}_{x} \mathrm{CoO}_{2}$ during discharging ${ }^{[36]}$; (d) in situ XRD during the first charge/discharge of $\mathrm{Na}_{x} \mathrm{CoO}_{2}{ }^{[59]}$

Reproduced with permission ${ }^{[58]}$, Copyright 2009 American Chemical Society. Reproduced with permission ${ }^{[36]}$, Copyright 2011 Nature Publishing group. Reproduced with permission ${ }^{[59]}$, Copyright 2014 Elsevier

$\mathrm{Na}^{+} /$空位的有序情况难以利用常见长程结构表征 技术，如 X 射线衍射进行直接分析. 固体核磁技术可通 过探测 $\mathrm{Na}_{x} \mathrm{CoO}_{2}$ 中 $\mathrm{Na}^{+}$的化学环境来推知局域结构情 况, 进而分析 $\mathrm{Na} /$ 空位的有序情况 ${ }^{[65-68]} .2015$ 年 Mukhamedshin 等 ${ }^{[69]}$ 总结了针对 $\mathrm{P} 2-\mathrm{Na}_{x} \mathrm{CoO}_{2}(0.65<x<$ 0.80 ) 的 NMR 研究 (如图 7b). 其中 $\mathrm{P} 2-\mathrm{Na}_{x} \mathrm{CoO}_{2}$ 的 ${ }^{23} \mathrm{Na}$-ssNMR 谱中的位于中心峰两旁的卫星峰发生了裂 分，表明 $\mathrm{P} 2-\mathrm{Na}_{x} \mathrm{CoO}_{2}$ 存在特定的 $\mathrm{Na}^{+}$排布情况.

此外, 研究者还通过电子衍射来观察 $\mathrm{Na}_{x} \mathrm{CoO}_{2}$ 中的 $\mathrm{Na}^{+}$-空位超晶格现象, 为 $\mathrm{Na}^{+}$-空位寻求证据. 2004 年 Zandbergen 等 ${ }^{[70]}$ 通过电子衍射研究了 $\mathrm{Na}_{x} \mathrm{CoO}_{2}(0.15<x$ $<0.75)$ 的结构特征, 在文献所报道的 $\mathrm{Na} 1$ 和 $\mathrm{Na} 2$ 位点分 布情况的基础上, 通过建模得出了不同 $\mathrm{Na}^{+}$含量下的 $\mathrm{Na}^{+} /$空位有序情况, 如图 7c 所示. 2008 年 Chou 等 ${ }^{[71]}$ 也 对 $\mathrm{Na}_{x} \mathrm{CoO}_{2}(x=0.84,0.71)$ 进行了研究, 利用 Roger 等 ${ }^{[72]}$ 提出的空位堆积模型分析了 $\mathrm{Na}^{+} /$空位有序情况. 其中 $\mathrm{Na}_{0.84} \mathrm{CoO}_{2}$ (图 7d(A C ) ) 的超晶格结构是以 $\mathrm{Na} 1$ 位点为 中心, 中心被 3 个空 $\mathrm{Na} 2$ 位点 (虚线圆圈)围绕; $\mathrm{Na}_{0.71} \mathrm{CoO}_{2}$ (图 7d(D F)) 的超晶格结构是以 $\mathrm{Na} 1$ 三聚体 中心, $\mathrm{Na} 1$ 位在 $\mathrm{A}$ 层中被三空位环绕, 在 $\mathrm{B}$ 层中被四空
位环绕. 同时, 拉曼光谱也是研究局域原子排列变化的 有效方法. 对于 $\mathrm{Na}_{x} \mathrm{CoO}_{2}$, 其在拉曼光谱中的 $\mathrm{E}_{2 \mathrm{~g}}$ 模式通 常因为 $\mathrm{Na}^{+}$的无序分布和高迁移率而消失, 这意味着 $\mathrm{E}_{2 \mathrm{~g}}$ 模式的出现可对应于 $\mathrm{Na}^{+}$排布的有序化. 2006 年 $\mathrm{Li}$ 等 ${ }^{[73]}$ 研究了 $\mathrm{Na}_{x} \mathrm{CoO}_{2}(x=0.7,0.5,0.3)$ 的拉曼光谱特征. 如图 7e 所示, $\mathrm{Na}_{0.7} \mathrm{CoO}_{2}$ 在室温下可观察到三个拉曼峰: $576 \mathrm{~cm}^{-1}$ 处的 $\mathrm{A}_{1 \mathrm{~g}}, a b$ 面 $460 \mathrm{~cm}^{-1}$ 处的 $\mathrm{E}_{2 \mathrm{~g}}$ 和 $a c$ 面 497 $\mathrm{cm}^{-1}$ 处的 $\mathrm{E}_{1 \mathrm{~g}}$. 对于 $\mathrm{Na}_{0.5} \mathrm{CoO}_{2}$, 在 $a b$ 面 $430 \mathrm{~cm}^{-1}$ 附近出 现了额外的一个 $\mathrm{E}_{2 \mathrm{~g}}$ 峰, 该峰在 $x=0.3$ 时消失. 上述谱 学特征意味着 $\mathrm{Na}_{x} \mathrm{CoO}_{2}$ 在 $x=0.7,0.5$ 时存在 $\mathrm{Na}^{+}$/空位有 序结构, 在 $x=0.3$ 时 $\mathrm{Na}^{+} /$空位转为无序分布, 这与固体 核磁技术与电子衍射表征得出的结果是基本一致的：即 当 $x$ 降低至 0.3 左右时, $\mathrm{Na}_{x} \mathrm{CoO}_{2}$ 中 $\mathrm{Na}^{+}$空位表现为无序 分布, 而当 $0.84>x>0.3$ 时, $\mathrm{Na}_{x} \mathrm{CoO}_{2}$ 保持有序分布. 其 中电子衍射与拉曼光谱表征中 $\mathrm{Na}_{x} \mathrm{CoO}_{2}$ 在 $x=0.3$ 与 $x=$ 0.33 的结果出现了差异, 这可能是由于文献在当年的合 成技术与条件下, 产物中钠量常具有的不确定性所导致 的.

对于电池的电化学性能而言, $\mathrm{Na}^{+}$空位有序性的存 在往往会限制 $\mathrm{Na}^{+}$的扩散，降低 $\mathrm{Na}^{+}$的利用率，进而对 


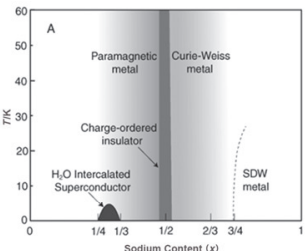

(a)
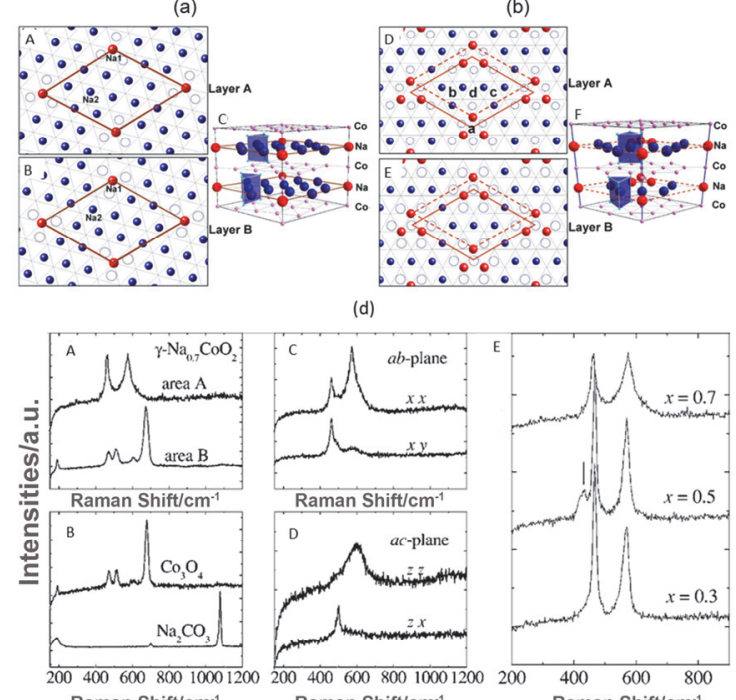

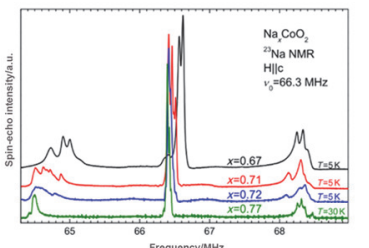

(b)

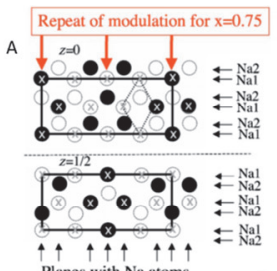

Planes with Na atoms
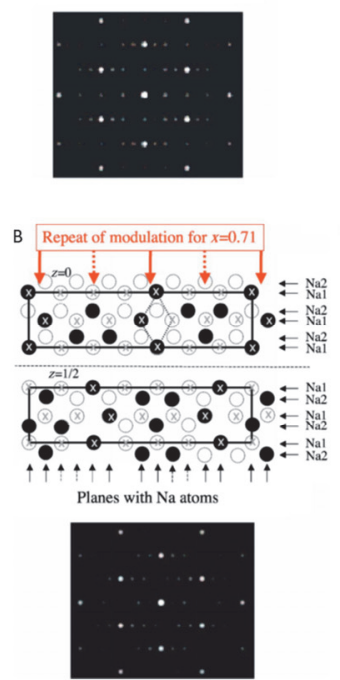

B Repeat of modulation for $x=0.71$
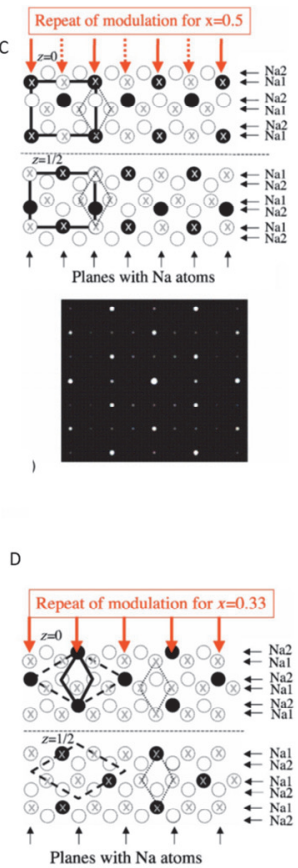

(c)
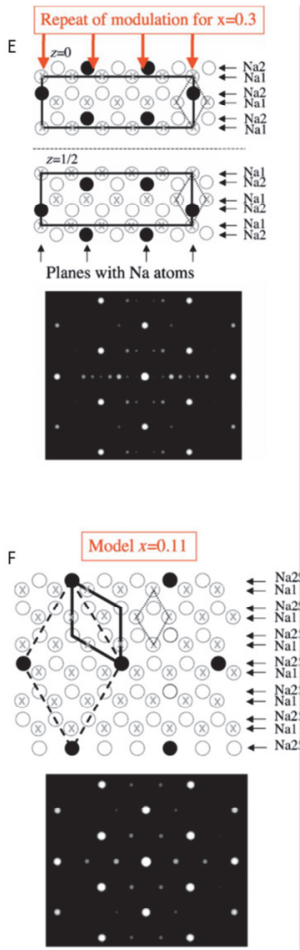

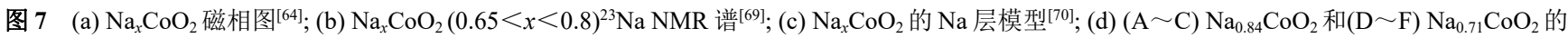
晶体结构 ${ }^{[71]}$; (e)室温下不同材料的拉曼光谱: (A) $\mathrm{Na}_{0.7} \mathrm{CoO}_{2},(\mathrm{~B}) \mathrm{Na}_{2} \mathrm{CO}_{3}$ 和 $\mathrm{Co}_{3} \mathrm{O}_{4},(\mathrm{C}) \mathrm{Na}_{0.7} \mathrm{CoO}_{2}$ 的 $a b$ 面, (D) $\mathrm{Na}_{0.7} \mathrm{CoO}$ 的 $a c$ 面, (E) $\mathrm{Na}_{x} \mathrm{CoO}$ ( $x=0.7$, $0.5,0.3)$ 的 $a b$ 面 ${ }^{[72]}$

Figure 7 (a) The phase diagram of $\mathrm{Na}_{x} \mathrm{CoO}_{2}{ }^{[64]}$; (b) ${ }^{23} \mathrm{Na} \mathrm{NMR}$ spectra for $\mathrm{Na}_{x} \mathrm{CoO}_{2}(0.65<x<0.8)^{[69]}$; (c) models for the $\mathrm{Na}$ planes of $\mathrm{Na} \mathrm{CoO}_{2}{ }^{[70]}$; (d) proposed crystal structure of (A-C) $\mathrm{Na}_{0.84} \mathrm{CoO}_{2}$ and (D-F) $\mathrm{Na}_{0.71} \mathrm{CoO}_{2}{ }^{[71]}$; (e) room-temperature Raman spectra of $(\mathrm{A}) \mathrm{Na}_{0.7} \mathrm{CoO}_{2},(\mathrm{~B}) \mathrm{Na}_{2} \mathrm{CO}_{3}$, and $\mathrm{Co}_{3} \mathrm{O}_{4}$ and polarized spectra from (C) $a b$ plane and (D) $a c$ plane of the $\mathrm{Na}_{0.7} \mathrm{CoO}_{2}$, (E) Raman spectra from the $a b$ surface of $\mathrm{Na}_{x} \mathrm{CoO} \mathrm{O}_{2}$ single crystals with $x=0.7$, 0.5 , and 0.3 at room temperature ${ }^{[72]}$

In (d), occupied Na positions are black dots and Na vacancies are open circles; $x$ indicates the positions of the underlying Co atoms; dashed lines indicate that only one of the lines on $z=0$ and $z=1 / 2$ is occupied; calculated diffraction patterns are given below the models. Reprinted figures with permission ${ }^{[64]}$, Copyright 2004 by the American Physical Society. Reproduced with permission ${ }^{[69]}$, Copyright 2015 Elsevier. Reprinted figures with permission ${ }^{[70]}$, Copyright 2004 by the American Physical Society. Reprinted figures with permission ${ }^{[71]}$, Copyright 2008 by the American Physical Society. Reprinted figures with permission ${ }^{[72]}$, Copyright 2006 by the American Physical Society

电池倍率性能造成不利影响. 研究发现可以通过掺杂电 化学惰性元素, 如 $\mathrm{Ca}^{2+}, \mathrm{Ti}^{4}$ 等可以有效抑制 $\mathrm{Na}^{+}$/空位 有序情况 ${ }^{[74]}$. 2015 年 $\mathrm{Han}$ 等 ${ }^{[43]}$ 对比了 $\mathrm{Na}_{0.60} \mathrm{Ca}_{0.07} \mathrm{CoO}_{2}$ 与 $\mathrm{Na}_{0.73} \mathrm{CoO}_{2}$ 的性能. 以 $235 \mathrm{~mA} / \mathrm{g}$ 电流密度进行充放电 时, 掺杂样品 $\mathrm{Na}_{0.60} \mathrm{Ca}_{0.07} \mathrm{CoO}_{2}$ 放电比容量为 $71 \mathrm{mAh} / \mathrm{g}$, 而 $\mathrm{Na}_{0.73} \mathrm{CoO}_{2}$ 为 $31 \mathrm{mAh} / \mathrm{g}$, 当随后电流密度恢复至 23.5 $\mathrm{mA} / \mathrm{g}$ 进行充放电时, 掺杂样品的容量恢复到 103 $\mathrm{mAh} / \mathrm{g}$, 且在随后循环中不出现明显的衰减, 但原始样 品则表现出明显容量衰减.

尽管固体核磁波谱、电子衍射、拉曼光谱对 $\mathrm{Na}_{x} \mathrm{CoO}_{2}$ 中 $\mathrm{Na}^{+} /$空位有序性问题给出了丰富的表征数据和解释 说法, 但到目前为止, $\mathrm{Na}^{+} /$空位有序情况是否会对层间 滑移的发生、 $\mathrm{Na}^{+}$脱嵌过程动力学有影响, 这些问题尚 无全面合理的解释, 这主要是由于对于 $\mathrm{Na}^{+} /$空位的有 序情况还没有可靠的表征手段. 虽然电子衍射技术可以 为该问题提供直接表征信息, 但在此基础上进行的 $\mathrm{Na}^{+} /$空位有序模型分析通常是基于一系列前提假设, 对 准确性有一定负面影响.

\section{4 钴酸钠电荷补偿机理研究进展}

对于 $\mathrm{Na}_{x} \mathrm{CoO}_{2}$, 如果假定为纯离子晶体场, 则在充 放电过程中 Co 的氧化态将从 III 变为 IV. 理想的电化学 脱嵌反应将是:

$$
\mathrm{Na}_{x} \mathrm{Co}^{I I I} \mathrm{O}_{2} \underset{\text { 放电 }}{\stackrel{\text { 充电 }}{\rightleftarrows}} \mathrm{Na}_{x-y} \mathrm{Co}^{I I I+x} \mathrm{O}_{2}+y \mathrm{Na}^{+}+y \mathrm{e}^{-}
$$

$X$ 射线光电子能谱(XPS)和 $X$ 射线吸收光谱(XAS) 均为直接表征元素价态的有效表征方法. 2013 年 $\mathrm{Fu}$ 等[39]通过非原位 XPS 和原位 XAS 表征了 $\mathrm{P} 2-\mathrm{Na}_{0.74} \mathrm{CoO}_{2}$ 的充放电的电荷补偿机制. 如图 $8 \mathrm{~b}$ 所示, XPS 谱中监测 到了 $\mathrm{Co}^{3+}$ 和 $\mathrm{Co}^{4^{+}}$的结合能变化, 证明了 $\mathrm{Na}^{+}$在层状结构 中的脱嵌伴随着 $\mathrm{Co}^{3+} / \mathrm{Co}^{4+}$ 氧化还原反应. $\mathrm{Co}^{3+} / \mathrm{Co}^{4^{+}}$的 最外层电子构型可以表示为 $\mathrm{t}^{6}{ }_{2 \mathrm{~g}} \mathrm{e}_{\mathrm{g}} \mathrm{t}^{5}{ }_{2 \mathrm{~g}} \mathrm{e}_{\mathrm{g}}{ }_{\mathrm{g}}$, 在充电过程 的氧化反应中变化仅发生在 $\mathrm{t}_{2 \mathrm{~g}}$ 简并轨道, 以至于 $\mathrm{Co}^{3^{+}} /$ $\mathrm{Co}^{4+}$ 中的能量变化非常小, 进而导致离子半径变化很 小. 基于同步辐射的原位吸收谱监测 $\mathrm{Na}^{+}$脱出过程(如 
图 $8 \mathrm{a}$ 所示)发现 $\mathrm{Co}-\mathrm{Co}$ 键长没有明显的变化, 这与 XPS 观察到的现象是吻合的, 该工作成功验证了 $\mathrm{Na}^{+}$在 钴酸钠的层状结构中的脱嵌过程中伴随着 $\mathrm{Co}^{3+} / \mathrm{Co}^{4+}$ 氧 化还原反应.

但通常在过渡金属 $(\mathrm{TM})$ 化合物中, TM-d 和 L-p $(\mathrm{L}$ 为 配体)轨道之间会发生一定程度的混合, 这使得 $\mathrm{TM}-\mathrm{L}$ 键具备共价性, 意味着在过渡金属化合物中, 配体氧也 可能参与电荷补偿过程. 对于三方 $D_{3 d}$ (立方 $O_{h}$ ) 对称晶 体中的 Co 和其他具有 $3 \mathrm{~d}$ 轨道的过渡金属氧化物, 轨道 混合主要发生在过渡金属离子 $3 \mathrm{~d}$ 的 $\mathrm{a}_{1 \mathrm{~g}}\left(\mathrm{t}_{2 \mathrm{~g}}\right)$ 轨道和氧的 $2 p$ 轨道之间 ${ }^{[75]}$. 因此, 在强氧化条件下, 只要 Co 的 $\mathrm{a}_{1 \mathrm{~g}}\left(\mathrm{t}_{2 \mathrm{~g}}\right)$ 轨道和氧的 $2 \mathrm{p}$ 轨道能量足够接近, $2 \mathrm{p}$ 轨道将补偿 $\mathrm{a}_{1 \mathrm{~g}}\left(\mathrm{t}_{2 \mathrm{~g}}\right)$ 轨道丢失的电子, 发生的氧化还原反应就不再是 从 $\mathrm{Co}^{\mathrm{III}}$ 到 $\mathrm{Co}^{\mathrm{IV}}$ 的简单变化.

对于氧是否参与电荷补偿这一问题, XPS 和 XAS 同 样可以给出有效表征信息. 2007 年 Valkeapaa 等[76]收集 了 $\mathrm{Na}_{x} \mathrm{CoO}_{2-\delta}(x=0.75,0.47,0.36,0.12)$ 样品的非原位 XAS 的 O $1 \mathrm{~s}$ 谱. 如图 $8 \mathrm{c}$ 所示, 每个光谱都具有两个特 征边: $530 \mathrm{eV}$ 附近的吸收边和 $534 \sim 548 \mathrm{eV}$ 之间的吸收 边, 分别对应于从 $\mathrm{O} 1 \mathrm{~s}$ 态过渡到 $\mathrm{O} 2 \mathrm{p}-\mathrm{Co} 3 \mathrm{~d}$ 和 O $2 \mathrm{p}-\mathrm{Co}$ $4 \mathrm{sp}$ 混合态的转变. 2021 年 Hausbrand 等 ${ }^{[40]}$ 对 $\mathrm{Na}_{x} \mathrm{CoO}_{2}$ 固态电池进行了原位 XPS 和 XAS 表征. 如图 $8 \mathrm{~d}(\mathrm{~A}, \mathrm{~B})$ 所示, 当材料充电时, $\mathrm{O} 1 \mathrm{~s}$ 谱中可以区分为两个高度混 合的成分：部分氧化的氧阴离子 $\mathrm{O}^{x-}\left(\mathrm{Co}_{\mathrm{h}}\right)$ 和氧化的氧二 聚体 $\left(\mathrm{O}_{2}\right)^{n-}$, 即证明了在 $\mathrm{Na}^{+}$脱嵌过程中存在氧参与的 混合氧化还原行为. 对于 $\mathrm{O} \mathrm{K}$ 边, 充电过程中存在四个
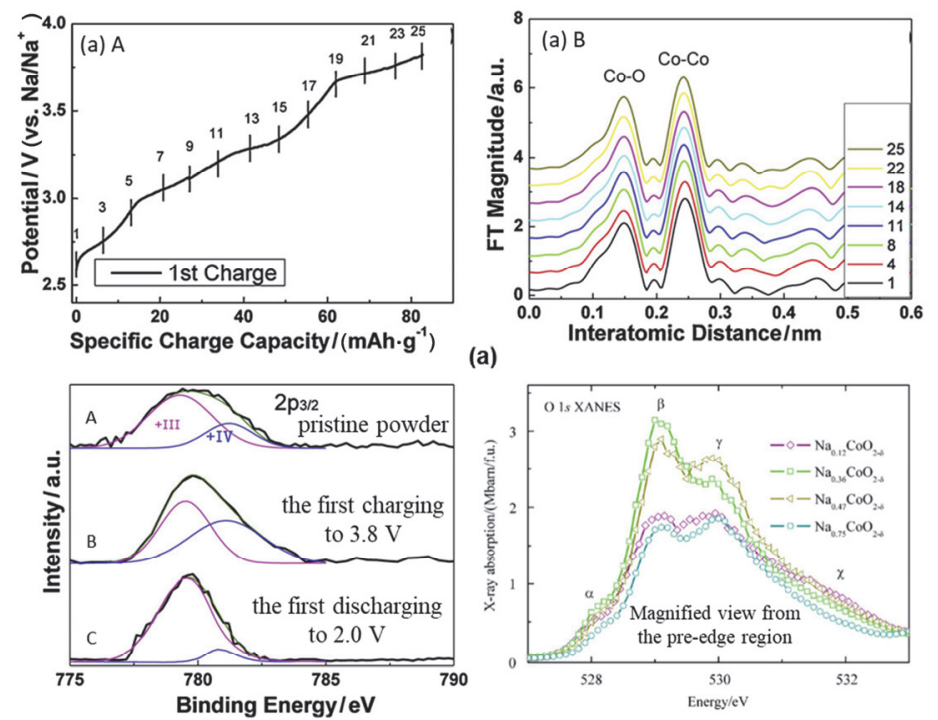

(b) (a)

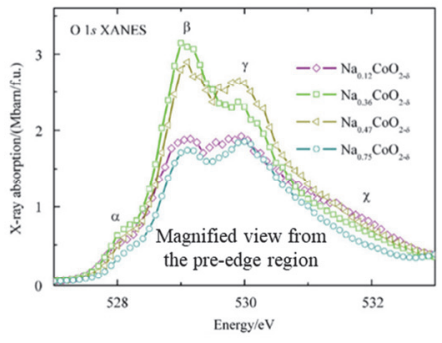

(c)
吸收边 I, II, III, IV, 分别对应于 $\mathrm{O} 2 \mathrm{p}^{-\mathrm{Co}^{4}} \mathrm{a}_{1 \mathrm{~g}}, \mathrm{O} 2 \mathrm{p}-$ $\mathrm{Co}^{4^{+}} \mathrm{e}_{\mathrm{g}}, \mathrm{O} 2 \mathrm{p}-\mathrm{Co}^{3^{+}} \mathrm{e}_{\mathrm{g}}$ 和过氧二聚体形成的过氧化物组 分. 这些与氧相关的特征吸收边表明, 氧将参与钴酸钠 的电荷补偿过程.

为了最大限度地利用正极材料的容量, 提高电池的 上限截止电压是一种极为直接的方法, 可以使电池实际 容量进一步接近理论容量上限. 但是, 随着截止电压的 提高，氧作为阴离子参与氧化还原反应的程度将会越来 越大，剧烈的晶格氧氧化还原反应可能进一步导致晶格 中氧的脱出，进一步破坏材料结构，造成电池性能的快 速衰退. 所以，提高电池截止电压的同时必须保证 $\mathrm{Na}^{+}$ 仍能进行可逆的脱嵌入反应，例如通过改性材料设计使 得晶格氧的氧化还原反应具有更好的可逆性, 减少电解 液在高电压下的分解副反应等，但这对于实现在材料局 域结构层次的精准调控提出了极高的要求. 由于钴酸钠 本身复杂的演化机理，多种反应机制的交织使得人们目 前对钴酸钠这一材料还缺乏深层次的理解, 这使得上述 改性设计的实现变得更加困难.

\section{5 总结与展望}

$\mathrm{Na}_{x} \mathrm{CoO}_{2}$ 作为层状钠离子过渡金属氧化物中的典型 结构, 多种晶体结构在电化学过程中都关联丰富的结构 演化信息, 且对其电化学性能的发挥有较大的影响. 其 中目前对于 $\mathrm{O} 3-\mathrm{Na}_{x} \mathrm{CoO}_{2}$ 充放电过程中的结构演化理解 已经比较明晰：在充放电过程中 $\mathrm{O} 3-\mathrm{Na}_{x} \mathrm{CoO}_{2}$ 将发生

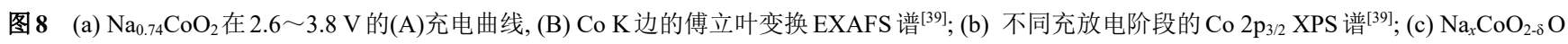
1s XANES 谱的放大视图 ${ }^{[76]}$; (d)不同充电阶段下 $\mathrm{Na}_{x} \mathrm{CoO}_{2-\delta}$ 的(A) O1s XPS 光谱, (B) XAS 的 $\mathrm{O} K$ 吸收边 ${ }^{[40]}$

Figure 8 (a) (A) The first charging curves of $\mathrm{Na}_{0.74} \mathrm{CoO}_{2}$ from 2.6 to $3.8 \mathrm{~V}$, (B) the Fourier transform of the Co K-edge EXAFS spectra ${ }^{[39]}$; (b) Co 2p $\mathrm{p}_{3 / 2}$ XPS spectra of different stages in charging and discharging processes ${ }^{[39]}$; (c) O 1s XANES spectra's magnified view of $\mathrm{Na}_{x} \mathrm{CoO}_{2-\delta}{ }^{[76]}$; (d) (A) O1s XPS spectra and (B) O K absorption edges for $\mathrm{Na}_{x} \mathrm{CoO}_{2-\delta}$ at different charge states ${ }^{[40]}$

Reproduced with permission ${ }^{[39]}$, Copyright 2013 Elsevier. Reproduced with permission ${ }^{[76]}$, Copyright 2007 Elsevier. Reproduced with permission ${ }^{[40]}$, Copyright 2021 Elsevier 
O3-O'3-P'3-P3-P'3 型相变, 由于 P'3 和 P3 相具有更快的 $\mathrm{Na}^{+}$扩散途径, 这使得该相变在一定程度上有利于材料 倍率性能的提升, 相变本身伴随的层间滑移过程将对循 环稳定性产生不利影响. $\mathrm{P} 2-\mathrm{Na}_{x} \mathrm{CoO}_{2}$ 具有更好的结构保 持能力, 在电化学过程中表现出更好的循环性能, 其在 低电压下的结构变化多为良性变化, 对电化学性能未造 成明显损害.

尽管如此, 目前关于 $\mathrm{P} 2-\mathrm{Na}_{x} \mathrm{CoO}_{2}$ 在充放电过程的 原位结构演化和机理解释还缺乏更加有效表征证据, 其 结构变化与电化学性能的构效关系尚未确立, 例如 $\mathrm{P} 2-\mathrm{Na}_{x} \mathrm{CoO}_{2}$ 电化学过程中原位晶胞参数演变的研究虽 已见报道, 但对于该材料在充放电过程中两相区域的指 认及是否存在新相等问题还未有深入研究.

P2 型钠离子层状过渡金属氧化物通常在不同的 $\mathrm{Na}^{+}$含量范围内表现出各种单相域, 这可能对应于不同 $\mathrm{Na}^{+}$/空位有序排布情况的超结构. 这种超结构的存在可 能对 $\mathrm{Na}^{+}$扩散等动力学参量造成影响. 但目前对于不同 $\mathrm{Na}^{+}$含量下 $\mathrm{Na}_{x} \mathrm{CoO}_{2}$ 结构中的 $\mathrm{Na}^{+}$/空位有序情况, 暂无 研究给出全面合理的表征和解释, 对层状材料中对 $\mathrm{Na}^{+} /$空位有序性的了解还十分局限. 一方面, 高时空分 辨的原位表征技术的进一步发展将有利于人们接近直 接观测上述有序排布的情况; 另一方面, 对 $\mathrm{Na}^{+} /$空位有 序排布的深入研究将有助于人们建立对电化学过程中 失效机制更加深入的理性认识. 有序排布等结构层面的 深入认识将有益于材料构效关系的建立并指导未来钠 离子电池高性能正极材料的设计. 例如, 通常认为 $\mathrm{Na}^{+} /$ 空位有序将抑制 $\mathrm{Na}^{+}$的扩散过程, 造成电池较差的倍率 性能表现. 目前已有报道通过掺杂电化学惰性元素来有 效抑制上述性能衰退, 也有报道 ${ }^{[77]}$ 通过合理的结构调 制策略进行 $\mathrm{Na}^{+} /$空位完全无序的材料设计, 这些经验 的累积都将有助于人们进一步设计并提升改进未来钠 离子电池层状正极材料的性能.

电荷补偿机制的研究往往针对阴阳离子两个组成 部分进行展开, 而目前针对包括钴酸钠在内的一些典型 材料的电荷补偿机制还并不明晰. 例如, 钴酸钠中的晶 格氧已经被证实将参与电化学氧化还原反应, 但随着电 位的增加, 剧烈氧反应的存在是否会导致钴酸钠材料中 晶格氧脱出? 失氧反应是否可逆? 是否将造成性能衰 退? 是否有可能调控氧反应来实现材料性能的改善? 针对典型材料展开电荷补偿相关失效机理的深入研究, 将为解决上述问题提供理论基础的指导和帮助.

和许多高容量的钠离子电池层状氧化物材料一样, 钴酸钠已被证实在充放电过程中同时存在结构变化和 电荷补偿这两大机制共同作用于电化学性能表现, 例如 目前关于钴酸钠中阴阳离子参与氧化还原反应的实验 论证已经相对完备. 但是, 目前钴酸钠的相关研究大多 停留在针对某一模块的独立机理研究或性能测试上. 一 方面, 钴酸钠材料中结构变化和电荷补偿机理与电化学
性能的关联性尚未直接建立，另一方面，在不同的电压 范围下进行的充放电过程中(例如调整上限电位等), 这 两大机制何者占主导地位还有待探寻, 这些问题的答案 将很大程度影响层状正极材料实际容量的充分发挥.

高性能电极材料设计的关键在于提升材料结构变 化与电荷补偿反应的可逆程度, 而性能的改进源于对材 料电化学过程的深刻理解和构效关系的指导. 未来若能 结合多种结构分析表征技术如高分辨透射电镜、固态核 磁及同步辐射光源等先进表征技术, 将为深入理解对应 固态电化学反应机理并推动建立材料构效关系提供重 要帮助. 而相应材料构效关系的建立，将有望为发展新 型高能量密度钠离子电池层状过渡金属正极材料提供 一份合理的设计原则.

\section{作者简介}

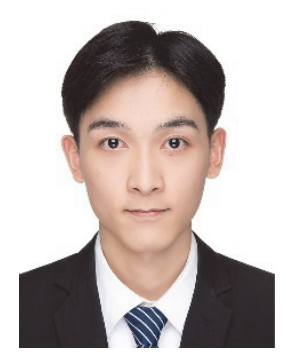

谢佶晟, 2021 年于厦门大学化学系获学士学位, 研究方向 为钠离子电池层状过渡金属氧化物正极材料的失效及改性机 理研究.

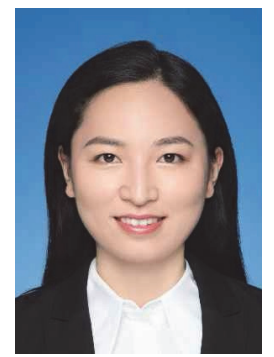

肖竹梅, 2018 年于南开大学化学系获学士学位, 现为厦门 大学化学化工学院杨勇教授课题组硕士研究生. 研究方向为 锂、钠离子电池中高比能正极材料的反应机理研究.

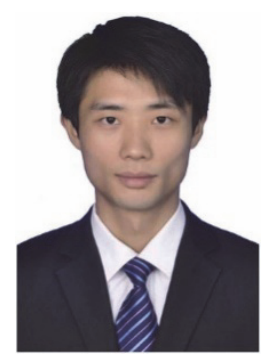

左文华, 2020 年于厦门大学获得博士学位, 师从杨勇教授, 目前主要从事钠离子电池层状过渡金属氧化物正极材料研究. 


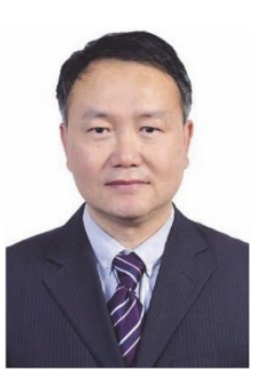

杨勇, 厦门大学闽江计划特聘教授, 博士生导师, 国家杰 出青年科学基金获得者. 1992 年获厦门大学理学博士学位, 1997 1998 年任英国牛津大学访问科学家. 主要研究方向为 能源电化学、材料物理化学及表面物理化学.

\section{References}

[1] Fang, Z.; Cao, Y.-L.; Hu, Y.-S.; Chen, L.-Q.; Huang, X.-J. Energy Storage Sci. Technol. 2016, 5, 149 (in Chinese). (方铮, 曹余良, 胡 勇胜, 陈立泉, 黄学杰, 储能科学与技术, 2016, 5,149 . )

[2] Christoph, V.; Daniel, B.; Marcel, W.; Stefano, P. Nat. Rev. Mater. 2018, 3, 1 .

[3] Li, H.; Wu, C.; Wu, F.; Bai, Y. Acta Chim. Sinica 2014, 72, 21 (in Chinese). (李慧, 吴川, 吴锋, 白莹, 化学学报, 2014, 72, 21.)

[4] Mizushima, K.; Jones, P. C.; Wiseman, P. J.; Goodenough, J. B. Solid State Ionics 1981, 3-4, 171.

[5] Kikkawa, S.; Miyazaki, S.; Koizumi, M. J. Solid State Chem. 1986, 62,35 .

[6] Li, W.; Liu, X.; Celio, H.; Smith, P.; Dolocan, A.; Chi, M.; Manthiram, A. Adv. Energy Mater. 2018, 8, 1703154.

[7] Noh, H.-J; Youn, S; Yoon, C. S; Sun, Y.-K. J. Power Sources 2013, $233,121$.

[8] Rossouw, M. H.; Thackeray, M. M. Mater. Res. Bull. 1991, 26, 463.

[9] Balsys, R. J.; Lindsay Davis, R. Solid State Ionics 1997, 93, 279.

[10] Delmas, C.; Braconnier, J.; Fouassier, C.; Hagenmuller, P. Solid State Ionics 1981, 3-4, 165.

[11] Molenda, J.; Delmas, C.; Hagenmuller, P. Solid State Ionics 1983, 9-10, 431 .

[12] Shacklette, L. W.; Jow, T. R.; Townsend, L. J. Electrochem. Soc. 1988, 135, 2669.

[13] Shacklette, L. W.; Jow, T. R.; Maxfield, M.; Hatami, R. Synthetic Met. 1989, 28, 655.

[14] Fouassier, C.; Matejka, G.; Reau, J.-M.; Hagenmuller, P. J. Solid State Chem. 1973, 6, 532.

[15] Liu, L.-L.; Qi, X.-G.; Hu, Y.-S.; Chen, L.-Q.; Huang, X.-J. Acta Chim. Sinica 2017, 75, 218 (in Chinese). (刘丽露, 戚兴国, 胡勇 胜, 陈立泉, 黄学杰, 化学学报, 2017, 75, 218.)

[16] Gutierrez, A.; Dose, W. M.; Borkiewicz, O.; Guo, F.; Avdeev, M.; Kim, S.; Fister, T. T.; Ren, Y.; Bareño, J.; Johnson, C. S. J. Phys. Chem. C 2018, 122, 23251.

[17] Lu, Z.; Dahn, J. R. J. Electrochem. Soc. 2001, 148, A1225.

[18] Yabuuchi, N.; Kajiyama, M.; Iwatate, J.; Nishikawa, H.; Shuji Hitomi; Ryoichi Okuyama; Ryo Usui; Yasuhiro Yamada; Shinichi Komaba. Nat. Mater. 2012, 11, 512.

[19] Wu, D.; Li, X.; Xu, B.; Twu, N.; Liu, L.; Ceder, G. Energy Environ. Sci. 2014, 8, 195.

[20] Kumakura, S.; Tahara, Y.; Sato, S.; Kubota, K.; Komaba, S. Chem. Mater. 2017, 29, 8958

[21] Didier, C.; Guignard, M.; Denage, C.; Szajwaj, O.; Ito, S.; Saadoune, I.; Darriet, J.; Delmas, C. Electrochem. Solid-State Lett. 2011, $14, \mathrm{~A} 75$.

[22] Yu, C.-Y.; Park, J.-S.; Jung, H.-G.; Chung, K.-Y.; Aurbach, D.; Sun, Y.-K.; Myung, S.-T. Energy Environ. Sci. 2015, 8, 2019.

[23] Caballero, A.; Hernán, L.; Morales, J.; Sánchez, L.; Santos Peña, J.; Aranda, M. A. G. J. Mater. Chem. 2002, 12, 1142.

[24] Mendiboure, A.; Delmas, C.; Hagenmuller, P. J. Solid State Chem. France 1985, 57, 323.

[25] Kikkawa, S.; Miyazaki, S.; Koizumi, M. Mater. Res. Bull. 1985, 20, 373.

[26] Wang, L.; Wang, J.; Zhang, X.; Ren, Y.; Zuo, P.; Yin, G.; Wang, J. Nano Energy 2017, 34, 215.
[27] Liu, X.; Zuo, W.; Zheng, B.; Xiang, Y.; Zhou, K.; Xiao, Z.; Shan, P.; Shi, J.; Li, Q.; Zhong, G.; Fu, R.; Yang, Y. Angew. Chem. Int. Ed. 2019, $58,18086$.

[28] Zuo, W.; Qiu, J.; Liu, X.; Ren, F.; Liu, H.; He, H.; Luo, C.; Li, J.; Ortiz, G. F.; Duan, H.; Liu, J.; Wang, M.-S.; Li, Y.; Fu, R.; Yang, Y. Nat. Commun. 2020, 11, 3544.

[29] Zuo, W.; Qiu, J.; Liu, X.; Zheng, B.; Zhao, Y.; Li, J.; He, H.; Zhou, K.; Xiao, Z.; Li, Q.; Ortiz, G. F.; Yang, Y. Energy Storage Mater. 2020, 26, 503.

[30] Zuo, W.; Ren, F.; Li, Q.; Wu, X.; Fang, F.; Yu, X.; Li, H.; Yang, Y. Nano Energy 2020, 78, 105285.

[31] Fang, Y.-J.; Chen, C.-X.; Ai, X.-P.; Yang, H.-X.; Cao, Y.-L. Acta Phys.-Chim. Sin. 2016, 33, 211 (in Chinese). (方永进, 陈重学, 艾 新平, 杨汉西, 曹余良, 物理化学学报, 2016, 33, 211.)

[32] Mizushima, K.; Jones, P. C.; Wiseman, P. J.; Goodenough, J. B. Mater. Res. Bull. 1980, 15, 783.

[33] Hertz, J. T.; Huang, Q.; McQueen, T.; Klimczuk, T.; Bos, J. W. G.; Viciu, L.; Cava, R. J. Phys. Rev. B 2008, 77, 75119.

[34] Wang, Z.; Wang, Z.; Peng, W.; Guo, H.; Li, X.; Wang, J.; Qi, A. Ionics 2014, 20, 1525.

[35] Takada, K.; Sakurai, H.; Takayama-Muromachi, E.; Izumi, F.; Dilanian, R. S. T. Nature 2003, 34, 53.

[36] Berthelot, R.; Carlier, D.; Delmas, C. Nat. Mater. 2011, 10, 74.

[37] Lei, Y.; Li, X.; Liu, L.; Ceder, G. Chem. Mater. 2014, 26, 5288.

[38] Terasaki, I.; Sasago, Y.; Uchinokura, K. Phys. Rev. B 1997, 56, 75397.

[39] Ding, J. J.; Zhou, Y. N.; Sun, Q.; Yu, X. Q.; Yang, X. Q.; Fu, Z. W. Electrochim. Acta 2013, 87, 388.

[40] Guhl, C.; Rohrer, J.; Kehne, P.; Ferber, T.; Alff, L.; Albe, K.; Jaegermann, W.; Komissinskiy, P.; Hausbrand, R. Energy Storage Mater. 2021, 37, 190.

[41] Delmas, C.; Fouassier, C.; Hagenmuller, P. Physica $B+C$ 1980, 99, 81.

[42] Blangero, M.; Carlier, D.; Pollet, M.; Darriet, J.; Delmas, C.; Doumerc, J.-P. Phys. Rev. B 2008, 77, 184116.

[43] Han, S. C.; Lim, H.; Jeong, J.; Ahn, D.; Park, W. B.; Sohn, K.-S.; Pyo, M. J. Power Sources 2015, 277, 9.

[44] Assadi, M. H. N.; Katayama-Yoshida, H. Comp. Mater. Sci. 2015, 109, 308.

[45] Bianchini, M.; Wang, J.; Clément, R.; Ceder, G. Adv. Energy Mater. 2018, 8,1801446 .

[46] Hasegawa, H.; Ishado, Y.; Okada, S.; Mizuhata, M.; Maki, H.; Matsui, M. J. Electrochem. Soc. 2021, 168, 10509.

[47] Yoshida, H.; Yabuuchi, N.; Komaba, S. Electrochem. Commun. 2013, 34, 60 .

[48] Kang, S. M.; Park, J.-H.; Jin, A.; Jung, Y. H.; Mun, J.; Sung, Y.-E. ACS Appl. Mater. Interfaces 2018, 10, 3562.

[49] Yabuuchi, N.; Kubota, K.; Dahbi, M.; Komaba, S. Chem. Rev. 2014, $114,11636$.

[50] Carlier, D.; van der Ven, A.; Delmas, C.; Ceder, G. Chem. Mater. 2003, 15, 2651.

[51] Liu, Y.-C.; Chen, C.-J.; Zhang, N.; Xiang, X.-D.; Chen, J. J. Electrochem. 2016, 22, 437 (in Chinese). (刘永畅, 陈程成, 张宁, 王刘 涁，向兴德，陈军，电化学, 2016, 22, 437.)

[52] Nayak, P. K.; Yang, L.; Brehm, W.; Adelhelm, P. Angew. Chem. Int. Ed. 2018, $57,102$.

[53] Biecher, Y.; Smiley, D. L.; Guignard, M.; Fauth, F.; Berthelot, R.; Delmas, C.; Goward, G. R.; Carlier, D. Inorg. Chem. 2020, 59, 5339.

[54] Liu, H.-Q.; Gao, X.; Chen, J.; Yin, S.-Y.; Zou, K.-Y.; Xu, L.-Q.; Zou, G.-Q.; Hou, H.-S.; Ji, X.-B. Energy Storage Sci. Technol. 2020, 9, 1327 (in Chinese). (刘欢庆, 高旭, 陈军, 尹首懿, 邹康宇, 徐来 强, 邹国强，侯红帅，纪效波，储能科学与技术, 2020, 9, 1327.)

[55] Zhu, X.-J.; Zhuang, Y.-H.; Zhao, Y.; Ni, M.-Z.; Xu, J.; Xia, H. Energy Storage Sci. Technol. 2020, 9, 1340 (in Chinese). (朱晓辉, 庄宇航，赵旸，倪明珠，徐璟，夏晖，储能科学与技术，2020，9, 1340.)

[56] Kubota, K.; Kumakura, S.; Yoda, Y.; Kuroki, K.; Komaba, S. Adv. Energy Mater. 2018, 8, 1703415.

[57] Yan, P.-F.; Zheng, J.-M.; Gu, M.; Xiao, J.; Zhang, J.-G.; Wang, C.-M. Nat. Commun. 2017, 8,1

[58] Carlier, D.; Blangero, M.; Ménétrier, M.; Pollet, M.; Doumerc, J.-P.; Delmas, C. Inorg. Chem. 2009, 48, 7018.

[59] Rai, A. K.; Anh, L. T.; Gim, J.; Mathew, V.; Kim, J. Ceram. Int. 2014, 40, 2411.

[60] Shu, G. J.; Chou, F. C. Phys. Rev. B 2008, 78. 
[61] Shibata, T.; Kobayashi, W.; Moritomo, Y. Appl. Phys. Express 2015, 8, 29202.

[62] Willis, T. J.; Porter, D. G.; Voneshen, D. J.; Uthayakumar, S.; Demmel, F.; Gutmann, M. J.; Roger, M.; Refson, K.; Goff, J. P. Sci. Rep. 2018, 8,3210 .

[63] Hilgenkamp, H.; Ariando.; Smilde, H.-J. H.; Blank, D. H. A.; Rijnders, G.; Rogalla, H.; Kirtley, J. R.; Tsuei, C. C. Nature 2003, 422, 50 .

[64] Foo, M. L.; Wang, Y.; Watauchi, S.; Zandbergen, H. W.; He, T.; Cava, R. J.; Ong, N. P. Phys. Rev. Lett. 2004, 92, 247001.

[65] Mukhamedshin, I. R.; Alloul, H.; Collin, G.; Blanchard, N. Phys. Rev. Lett. 2004, 93, 167601.

[66] Mukhamedshin, I. R.; Dooglav, A. V.; Krivenko, S. A.; Alloul, H. Phys. Rev. B 2014, 90, 115151.

[67] Platova, T. A.; Mukhamedshin, I. R.; Alloul, H.; Dooglav, A. V.; Collin, G. Phys. Rev. B 2009, 80, 224106.

[68] Platova, T. A.; Mukhamedshin, I. R.; Dooglav, A. V.; Alloul, H. JETP Lett. 2010, 91, 421.

[69] Mukhamedshin, I. R.; Alloul, H. Physica B: Condensed Matter 2015, 460, 58 .
[70] Zandbergen, H. W.; Foo, M.; Xu, Q.; Kumar, V.; Cava, R. J. Phys. Rev. B 2004, 70, 24101 .

[71] Chou, F. C.; Chu, M.-W.; Shu, G. J.; Huang, F.-T.; Pai, W. W.; Sheu, H. S.; Lee, P. A. Phys. Rev. Lett. 2008, 101, 127404.

[72] Roger, M.; Morris, D. J. P.; Tennant, D. A.; Gutmann, M. J.; Goff, J. P.; Hoffmann, J.-U.; Feyerherm, R.; Dudzik, E.; Prabhakaran, D.; Boothroyd, A. T.; Shannon, N.; Lake, B.; Deen, P. P. Nature 2007, 445, 631 .

[73] Qu, J. F.; Wang, W.; Chen, Y.; Li, G.; Li, X. G. Phys. Rev. B 2006, $73,250$.

[74] Chen, J. Acta Phys.-Chim. Sin. 2018, 35, 347 (in Chinese). (陈军, 物理化学学报, 2018, 35, 347.)

[75] de Groot, F. M. F.; Grioni, M.; Fuggle, J. C.; Ghijsen, J.; Sawatzky, G. A.; Petersen, H. Phys. Rev. B 1989, 40, 5715.

[76] Valkeapaa, M.; Katsumata, Y.; Asako, I.; Motohashi, T.; Chan, T. S.; Liu, R. S.; Chen, J. M.; Yamauchi, H.; Karppinen, M. J. Solid State Chem. 2007, 180, 1608.

[77] Wang, P.-F.; Yao, H.-R.; Liu, X.-Y.; Yin, Y.-X.; Zhang, J.-N.; Wen, Y.; Yu, X.; Gu, L.; Guo, Y.-G. Sci. Adv. 2018, 4, eaar6018.

(Cheng, B. 